\title{
The non-resonant response of fluid in a rapidly rotating sphere undergoing longitudinal libration
}

\author{
Keke Zhang ${ }^{1, \dagger}$, Kit H. Chan ${ }^{2}$, Xinhao Liao ${ }^{3}$ and Jonathan M. Aurnou \\ ${ }^{1}$ Department of Mathematical Sciences, University of Exeter, Exeter EX4 4QF, UK \\ ${ }^{2}$ Department of Mathematics, University of Hong Kong, Pokfulam, Hong Kong \\ ${ }^{3}$ Shanghai Astronomical Observatory, Chinese Academy of Sciences, Shanghai 200030, China \\ ${ }^{4}$ Department of Earth and Space Sciences, University of California, Los Angeles, CA 90095-1567, USA
}

(Received 11 August 2012; revised 22 December 2012; accepted 15 January 2013; first published online 27 February 2013)

We investigate the problem of oscillatory flow of a homogeneous fluid with viscosity $v$ in a fluid-filled sphere of radius $a$ that rotates rapidly about a fixed axis with angular velocity $\Omega_{0}$ and that undergoes weak longitudinal libration with amplitude $\epsilon \Omega_{0}$ and frequency $\hat{\omega} \Omega_{0}$, where $\epsilon$ is the Poincare number with $\epsilon \ll 1$ and $\hat{\omega}$ is dimensionless frequency with $0<\hat{\omega}<2$. Three different methods are employed in this investigation: (i) asymptotic analysis at small Ekman numbers $E$ defined as $E=v /\left(a^{2} \Omega_{0}\right)$; (ii) linear numerical analysis using a spectral method; and (iii) nonlinear direct numerical simulation using a finite-element method. A satisfactory agreement among the three different sets of solutions is achieved when $E \leqslant 10^{-4}$. It is shown that the flow amplitude $|\boldsymbol{u}|$ is nearly independent of both the Ekman number $E$ and the libration frequency $\hat{\omega}$, always obeying the asymptotic scaling $|\boldsymbol{u}|=O(\epsilon)$ even though various spherical inertial modes are excited by longitudinal libration at different libration frequencies $\hat{\omega}$. Consequently, resonances do not occur in this system even when $\hat{\omega}$ is at the characteristic value of an inertial mode. It is also shown that the pressure difference along the axis of rotation is anomalous: this quantity reaches a sharp peak when $\hat{\omega}$ approaches a characteristic value. In contrast, the pressure difference measured at other places in the sphere, such as in the equatorial plane, and the volume-integrated kinetic energy are nearly independent of both the Ekman number $E$ and the libration frequency $\hat{\omega}$. Absence of resonances in a fluid-filled sphere forced by longitudinal libration is explained through the special properties of the analytical solution that satisfies the no-slip boundary condition and is valid for $E \ll 1$ and $\epsilon \ll 1$.

Key words: geophysical and geological flows, rotating flows

\section{Introduction}

Gravitational interaction between a non-spherical planet and its parent star or moons results in non-uniform rotation of the planet that produces a Poincaré force driving fluid motion in the interior of the planet (see, for example, Dermott 1979; Tilgner 2007). For many planets, the non-uniform component of its angular velocity is usually 
small and, hence, the corresponding Poincaré force would also be weak (for example, Margot et al. 2007; Noir et al. 2009). As a consequence, fluid motion in the interior of a non-uniformly rotating planet forced by the Poincaré force would be weak and, hence, insignificant. However, it would become very strong and, hence, highly significant if the weak Poincare force resonates directly with any inertial modes, which are the eigenfunctions of a rapidly rotating fluid system.

The significance of resonance may be illustrated by looking at some general properties of non-uniform-rotation-driven flow $\boldsymbol{u}$. Suppose that a fluid-filled, axisymmetric container rotates rapidly but slightly non-uniformly in the form of precession or libration or both. In a rotating frame of reference attached to the container (Greenspan 1968; Tilgner 2007), which we shall adopt throughout this paper, fluid motion driven by weak non-uniform rotation is governed by the dimensionless equations of motion and continuity:

$$
\begin{gathered}
\frac{\partial \boldsymbol{u}}{\partial t}+2 \hat{z} \times \boldsymbol{u}+\nabla p=E \nabla^{2} \boldsymbol{u}+\epsilon\left(\frac{\partial \widehat{\boldsymbol{\Omega}}}{\partial t} \times \boldsymbol{r}\right), \\
\nabla \cdot \boldsymbol{u}=0,
\end{gathered}
$$

where $\boldsymbol{r}$ is the position vector, $p$ is a reduced pressure, $\boldsymbol{u}$ is the fluid velocity, $\widehat{\boldsymbol{\Omega}}$ is the dimensionless angular velocity which is time-dependent with $|\widehat{\boldsymbol{\Omega}}|=O(1), \hat{z}$ is a unit vector parallel to the axis of the primary rotation, $E$ is the Ekman number with $E \ll 1$ and $\epsilon$ is often referred to as the Poincaré number with $\epsilon \ll 1$.

In order to understand both the stability of spinning spacecraft with fluid payloads and planetary precession, the most extensively studied problem of non-uniformrotation-driven flow is concerned with fluid motion in a precessing cylinder (see, for example, Wood 1966; Gans 1970; Manasseh 1992; Kobine 1995; Meunier et al. 2008; Liao \& Zhang 2012). We use the problem of fluid motion in a precessing cylinder to illustrate the fundamental differences between the resonant and non-resonant responses in non-uniformly rotating fluids. When the excitation frequency $\hat{\omega}$ is at a characteristic value $\omega_{n k}$ of a cylindrical inertial mode $\boldsymbol{u}_{n k}$ and the Poincaré force $\epsilon[(\partial \hat{\boldsymbol{\Omega}} / \partial t) \times \boldsymbol{r}]$ is not orthogonal with the mode $\boldsymbol{u}_{n k}$ (i.e. $\int_{V}[(\partial \hat{\boldsymbol{\Omega}} / \partial t) \times \boldsymbol{r}] \cdot \boldsymbol{u}_{n k} \mathrm{~d} V \neq 0$ where $\int_{V} \mathrm{~d} V$ denoting the integration over the cylinder), resonances take place and are marked by the properties that the amplitude of the precessing flow $|\boldsymbol{u}|$ reaches a sharp peak and obeys the asymptotic scaling (for example, Gans 1970; Manasseh 1992; Meunier et al. 2008)

$$
|\boldsymbol{u}|=O(\epsilon / \sqrt{E}) \quad \text { for } E \ll 1 \text { at resonances. }
$$

At non-resonances, however, the flow amplitude is characterized by the asymptotic scaling (see, for example, Wood 1966; Kobine 1995; Meunier et al. 2008)

$$
|\boldsymbol{u}|=O(\epsilon) \quad \text { for } E \ll 1 \text { at non-resonances. }
$$

The above asymptotic scalings at $E \ll 1$ can be, in fact, deduced from the governing equation (1.1) without having to solve the complicated mathematical problem. For simplicity, let the Poincaré force $\epsilon[(\partial \hat{\Omega} / \partial t) \times \boldsymbol{r}]=\epsilon \boldsymbol{f}(\boldsymbol{r}) \mathrm{e}^{\mathrm{i} \hat{\omega} t}$ with $\mathrm{i}=\sqrt{-1}$ and $|\boldsymbol{f}|=O(1)$, and let the fluid response $\boldsymbol{u}=\mathscr{A} \boldsymbol{u}_{n k} \mathrm{e}^{\mathrm{i} \hat{\omega} t}$ by assuming that a single inertial mode $\boldsymbol{u}_{n k}$ with amplitude $\mathscr{A}$ would be excited either resonantly or non-resonantly. At non-resonances, the Poincaré force $\epsilon \boldsymbol{f}$ directly drives a weak flow $\mathscr{A}=O(\epsilon)$, as clearly seen from (1.1), for which the viscous effect in connection with $E \nabla^{2} \boldsymbol{u}$ is passive and plays a non-essential role at $E \ll 1$. At resonance, when $\hat{\omega}$ is at 
a characteristic value $\omega_{n k}$ of a cylindrical inertial mode and the Poincare force $\epsilon \boldsymbol{f}$ resonates with $\boldsymbol{u}_{n k}$, the dynamics becomes more complicated. In this case, the Poincaré force $\epsilon \boldsymbol{f}$ drives a time-dependent flow whose growing amplitude must be contained by active viscous effects that take place mainly in the Ekman boundary layer whose thickness is $O(\sqrt{E})$ and whose flux into the bulk fluid is $O(\mathscr{A} \sqrt{E})$. Equation (1.1) then leads to a balance between the flux momentum from the boundary layer into the bulk fluid and the Poincaré force

$$
\mathscr{A} \sqrt{E} \sim \epsilon|\boldsymbol{f}| \quad \text { at } E \ll 1,
$$

which gives rise to the resonant scaling $|\mathscr{A}|=O(\epsilon / \sqrt{E}$ ) (see, for example, Gans 1970; Meunier et al. 2008; Liao \& Zhang 2012).

In an important and highly influential work of rotating fluid experiments, Aldridge \& Toomre (1969) studied the problem of flow in a fluid-filled sphere forced by weak longitudinal libration. They considered a homogeneous fluid of viscosity $v$ in a sphere of radius $a$ that rotates rapidly about a fixed axis with the primary angular velocity $\Omega_{0} \hat{z}$ and, at the same time, undergoes weak longitudinal libration with the amplitude $\epsilon \Omega_{0}$ and the frequency $\hat{\omega} \Omega_{0}$, where $\epsilon \ll 1$ and $\hat{\omega}$ is the dimensionless frequency with $0<\hat{\omega}<2$. The instantaneous or overall angular velocity $\Omega$ of the spherical container is of the form

$$
\boldsymbol{\Omega}=\hat{z} \Omega_{0}\left[1+\epsilon \sin \left(\hat{\omega} \Omega_{0} t\right)\right],
$$

where $\Omega_{0}$ is the mean rate of rotation and $\hat{z}$ is a unit vector in the direction of rotation. Owing to the non-uniform rotation, the Poincare force $[(\partial \Omega / \partial t) \times \boldsymbol{r}]$ drives an oscillatory fluid motion in the fluid-filled sphere. In their laboratory experiments, Aldridge \& Toomre (1969) measured the dynamical response of the fluid in the form of pressure variation along the rotation axis to various libration frequencies $\hat{\omega}$. They demonstrated that the axisymmetric spherical inertial mode, which is described by the eigenfrequency $\omega_{n k}$, the eigenfunction velocity $\boldsymbol{u}_{n k}$ and the pressure $p_{n k}$ with $n$ being the axial wavenumber and $k$ the radial wavenumber, can be excited when the libration frequency $\hat{\omega} \rightarrow \omega_{n k}$. This excitation is clearly reflected in the measurement that the pressure difference amplitude along the axis of rotation reaches a sharp peak when the libration frequency $\hat{\omega}$ approaches the eigenfrequency $\omega_{n k}$ (see figures 3 and 7 of Aldridge \& Toomre 1969). This well-known experimental observation was interpreted theoretically, like that in a precessing cylinder, as being resonance with the inertial mode $\boldsymbol{u}_{n k}$ when $\hat{\omega}=\omega_{n k}$ (Greenspan 1968).

For understanding the physical implication of librationally driven flow in planets and moons, this classical problem, or an extension of the problem, has been also studied by various authors. Rieutord (1991), Tilgner (1999) and Calkins et al. (2010) studied numerically the response to longitudinal libration in spherical systems in which coupling between the librating container and its interior fluid is purely viscous. In particular, Rieutord (1991) showed that the pressure variation along the axis of rotation reaches a peak when the libration frequency approaches to the frequency of an inertial mode $\omega_{n k}$, with a focus on how a sizeable inner core in spherical shells may alter the pressure difference on the rotation axis. Recently, Zhang, Chan \& Liao (2012) showed that resonance can take place in a latitudinally librating spheroidal cavity. The amplitude of the librating flow is $|\boldsymbol{u}|=O(\epsilon / \sqrt{E})$ at $E \ll 1$ when the libration frequency $\hat{\omega}$ is at the characteristic value $\omega_{n k}$ of a particular inertial mode while $|\boldsymbol{u}|=O(\epsilon)$ away from resonance. Note that the form of the Poincare forcing, $\epsilon(\partial \widehat{\boldsymbol{\Omega}} / \partial t \times \boldsymbol{r})$, is different between the longitudinal and latitudinal libration. 
Experimental and numerical studies have also focused on the strongly nonlinear properties of longitudinally libration-driven flow when the Poincaré number $\epsilon$ becomes sufficiently large (Noir et al. 2009) or when the containers are non-axisymmetric and, hence, the topographical coupling between the container and fluid becomes significant (Chan, Liao \& Zhang 2011; Zhang, Chan \& Liao 2011; Cébron et al. 2012; Noir et al. 2012). In particular, Cébron et al. (2012) shows that the nonlinear flow, due to the topographical effect of non-axisymmetric geometry, may generate elliptical instabilities through the nonlinear interaction among different inertial modes. Moreover, Busse (2010) and Calkins et al. (2010) showed that longitudinal libration can drive a weak mean flow in librating spheres, which is confirmed by in the laboratory experiments of Noir et al. $(2010,2012)$ and Sauret et al. (2010). It is of significance to note that the responses in axisymmetric containers did not correspond to that of a global scale resonance with strong velocities being excited throughout the fluid shell (Noir et al. 2012). Thus, a significant problem exists in relating the interpretation of Aldridge \& Toomre (1969) to the results in more recent studies.

In the present study, we revisit the problem of fluid oscillation in a fluid-filled sphere forced by weak longitudinal libration. Three different but complementary approaches, asymptotic analysis at $E \ll 1$, linear numerical analysis and nonlinear direct numerical simulation, are adopted. The primary objective of this study is to clarify the two important questions of the problem. Does resonance, like that in a precessing cylinder or a latitudinally librating spheroid, occur in longitudinally librating spheres when the libration frequency $\hat{\omega}$ is at the characteristic value $\omega_{n k}$ of a spherical inertial mode? If the resonance cannot occur, then what is the implication of a sharp peak for the pressure difference amplitude measured along the axis of rotation at $\hat{\omega}=\omega_{n k}$ ? We shall show that the flow amplitude $|\boldsymbol{u}|$ in longitudinally librating spheres always obeys the asymptotic scaling $|\boldsymbol{u}|=O(\epsilon)$ at $E \ll 1$ at or near or away from a characteristic value $\omega_{n k}$, indicating the absence of resonance in this problem. We show that although the pressure difference along the axis of rotation attains a sharp peak when $\hat{\omega} \rightarrow \omega_{n k}$, the pressure variation at other places, such as in the equatorial plane, is nearly independent of both the Ekman number $E$ and the libration frequency $\hat{\omega}$. We also develop an asymptotic theory that helps explain the result of the linear and nonlinear numerical analysis.

In what follows we shall begin by presenting the mathematical formulation of the problem in $\S 2$. An asymptotic solution at $\hat{\omega}=\omega_{n k}$ for $E \ll 1$ is derived in $\S 3$, providing the mathematical reasoning of why resonance cannot occur in the present problem. Linear numerical analysis using a spectral method is discussed in $\S 4$ while nonlinear direct numerical simulation using a finite-element method is presented in $\S 5$. A comparison among the three different sets of results is presented in $\S 6$. Finally, a brief summary and concluding remarks are given in $\S 7$.

\section{Mathematical formulation}

Consider a viscous, homogeneous fluid of viscosity $v$ and density $\rho$ confined in a spherical cavity. Suppose that the spherical container rotates rapidly with a nonuniform angular velocity $\Omega$ given by (1.6). In a frame of reference attached to the container, librationally driven flow in the spherical cavity is governed by the 
dimensional equations (see, for example, Greenspan 1968):

$$
\begin{gathered}
\frac{\partial \boldsymbol{u}}{\partial t}+\boldsymbol{u} \cdot \nabla \boldsymbol{u}+2 \Omega_{0}\left(1+\epsilon \sin \hat{\omega} \Omega_{0} t\right) \hat{z} \times \boldsymbol{u}+\frac{1}{\rho} \nabla p \\
=v \nabla^{2} \boldsymbol{u}+\boldsymbol{r} \times \frac{\mathrm{d}}{\mathrm{d} t}\left[\Omega_{0}\left(1+\epsilon \sin \hat{\omega} \Omega_{0} t\right) \hat{z}\right], \\
\nabla \cdot \boldsymbol{u}=0,
\end{gathered}
$$

where $\epsilon \ll 1, \boldsymbol{u}$ is the three-dimensional velocity field $\boldsymbol{u}=\left(u_{r}, u_{\theta}, u_{\phi}\right)$, with corresponding unit vectors $(\hat{\boldsymbol{r}}, \hat{\boldsymbol{\theta}}, \hat{\boldsymbol{\phi}})$, in spherical polar coordinates $(r, \theta, \phi)$ with $\theta=0$ at the axis of $\hat{z}$ and $r=0$ at the centre of the sphere. The centrifugal force is combined with other conservative forces to form the reduced pressure $p$. The final term on the right-hand side of (2.1) is known as the Poincaré force which drives librational flow against viscous dissipation. The libration amplitude $\Omega_{0} \epsilon$ controls the degree of nonlinearity of the problem and will be assumed to be small.

Employing the radius of the sphere $a$ as the length scale, $\Omega_{0}^{-1}$ as the unit of time and $\rho a^{2} \Omega_{0}^{2}$ as the unit of pressure yields the dimensionless equations:

$$
\begin{gathered}
\frac{\partial \boldsymbol{u}}{\partial t}+\boldsymbol{u} \cdot \nabla \boldsymbol{u}+2(1+\epsilon \sin \hat{\omega} t) \hat{z} \times \boldsymbol{u} \\
\boldsymbol{\nabla} \cdot \boldsymbol{u}=0, E \nabla^{2} \boldsymbol{u}+\epsilon \hat{\omega}(\boldsymbol{r} \times \hat{z}) \cos (\hat{\omega} t),
\end{gathered}
$$

where the Ekman number, $E=v / \Omega_{0} a^{2}$, provides the measure of relative importance between the typical viscous force and the Coriolis force, and the Poincare number $\epsilon$ quantifies the strength of the Poincaré forcing. Librationally driven flow on the bounding spherical surface, $\mathscr{S}$, of the container is at rest, requiring that

$$
\hat{\boldsymbol{r}} \cdot \boldsymbol{u}=0 \text { and } \hat{\boldsymbol{r}} \times \boldsymbol{u}=\mathbf{0} \text { at } r=1 .
$$

When $\epsilon$ is sufficiently small with $|\boldsymbol{u}|=O(\delta) \ll 1$, (2.3) may be linearized by omitting the square or products of small terms, $\boldsymbol{u} \cdot \nabla \boldsymbol{u}=O\left(\delta^{2}\right)$ and

$$
|\epsilon \sin (\hat{\omega} t) \hat{z} \times \boldsymbol{u}|=O(\delta \epsilon),
$$

which yields the governing equation for weak libration

$$
\begin{aligned}
\frac{\partial \boldsymbol{u}}{\partial t}+2 \hat{\boldsymbol{z}} \times \boldsymbol{u} & =-\nabla p+E \nabla^{2} \boldsymbol{u}+\epsilon \hat{\omega}(\boldsymbol{r} \times \hat{\boldsymbol{z}}) \mathrm{e}^{\mathrm{i} \hat{\omega} t}, \\
\nabla \cdot \boldsymbol{u} & =0,
\end{aligned}
$$

where only the real part of the complex solution is taken as the physical solution.

The weakly librating flow $(\epsilon \ll 1)$, defined by $(2.7)-(2.8)$ subject to the noslip boundary condition (2.5), is first solved asymptotically for $E \ll 1$ and, then, numerically using a spectral method. To validate the linear approximation and compute the pressure field directly, the fully nonlinear problem, defined by (2.3)-(2.4) subject to the boundary condition (2.5), is also solved by numerical simulation using a finiteelement method.

\section{Asymptotic analysis}

The existing theory of the problem was based on the pressure expansion (see (6.2)-(6.4) in $\S 6$ of Greenspan (1964) and (2.14.5)-(2.14.8) in Greenspan (1968)). Consequently, the no-slip velocity condition cannot be explicitly enforced. Owing to the appearance of the singular terms such as $1 /\left(\hat{\omega}-\omega_{n k}\right)$ in the pressure-expansion 
theory, it suggests that resonance with the inertial mode $\boldsymbol{u}_{n k}$ would take place as the libration frequency $\hat{\omega}$ approaches the characteristic value $\omega_{n k}$ of the inertial mode.

With the availability of the explicit expression for both the velocity $\boldsymbol{u}_{n k}$ and the pressure $p_{n k}$ for any spherical inertial modes (Zhang et al. 2001), our asymptotic analysis will be based on the velocity-pressure expansion such that the no-slip condition can be explicitly enforced. In the present velocity-pressure formulation, it is the resulting solvability condition, which is explicitly independent of $E$ without having any singular terms such as $1 /\left(\hat{\omega}-\omega_{n k}\right)$, that will determine the amplitude of a librating flow at $\hat{\omega}=\omega_{n k}$. We shall start with a brief argument of why resonance mathematically cannot occur in the present libration problem.

\subsection{Why resonance cannot occur}

A subset of the spherical inertial modes which are azimuthally axisymmetric (because of spherical geometry) and equatorially symmetric may be excited by the Poincaré force $(\epsilon \hat{\omega})(\boldsymbol{r} \times \hat{\boldsymbol{z}}) \cos (\hat{\omega} t)$. Any axisymmetric mode in this subset is described by its velocity $\boldsymbol{u}_{n k}$, the pressure $p_{n k}$ and the eigenfrequency $\omega_{n k}$. According to Zhang et al. (2001), the pressure $p_{n k}$ for the subset is expressible as

$$
p_{n k}(r, \theta)=\sum_{i=0}^{k} \sum_{j=0}^{k-i} \mathscr{C}_{k i j} r^{2(i+j)} \sigma_{n k}^{2 i}\left(1-\sigma_{n k}^{2}\right)^{j} \sin ^{2 j} \theta \cos ^{2 i} \theta,
$$

for $k=2,3,4, \ldots$, where the term with $i+j=0$ should be excluded, and $\mathscr{C}_{k i j}$ is defined as

$$
\mathscr{C}_{k i j}=\frac{(-1)^{i+j}[2(k+i+j)-1] ! !}{2^{j+1}(2 i-1) ! !(k-i-j) ! i !(j !)^{2}},
$$

along with

$$
(2 j-1) ! !=(2 j-1) \ldots(3)(1), \quad(-1) ! !=1, \quad 0 !=1 .
$$

An arbitrary normalization is used to keep the expression as simple as possible. In (3.1), the half frequency $\sigma_{n k}$, i.e. $\sigma_{n k}=\omega_{n k} / 2$, is the solution of

$$
\sum_{j=0}^{k-1}\left\{\frac{(-1)^{j}[2(2 k-j)] !}{j ![2(k-j)-1] !(2 k-j) !}\right\} \sigma_{n k}^{2(k-j)}=0,
$$

where $k$ varies over all positive integers with $k \geqslant 2$. There exist $2(k-1)$ distinct real roots to (3.4) within $-1<\sigma_{n k}<1$. Since it is $\sigma_{n k}^{2}$ that appears in (3.4), we shall always have a pair of $+\left|\sigma_{n k}\right|$ and $-\left|\sigma_{n k}\right|$ as solutions to (3.4). Therefore, it suffices to focus on only the inertial modes with $+\left|\sigma_{n k}\right|$. Different positive roots of (3.4) can be arranged, with the aid of subscript notation, according to the size of $\sigma_{n k}$,

$$
0<\sigma_{1 k}<\sigma_{2 k}<\sigma_{3 k}, \ldots,<\sigma_{n k}<\cdots,
$$

where $n$, the first index, is restricted by $n \leqslant(k-1)$, and $\sigma_{n k}$ denotes the $n$th positive smallest root to (3.4) for given $k$. The first fundamental inertial mode having the simplest spatial structure is given by $k=2$ and $n=1$. Note that the index notation used in Aldridge \& Toomre (1969) is slightly different: our $\omega_{n k}$ corresponds to their $\omega_{(k-1) n}$. For example, the three fundamental inertial modes in our notation are $\omega_{12}, \omega_{13}$ and $\omega_{23}$ which in their notion are $\omega_{11}, \omega_{21}$ and $\omega_{22}$. Physically, the subscript $n$ represents roughly the degree of the complexity in the axial direction 
along the rotation axis while the index $k$ reflects the radial structure in the direction perpendicular to the rotation axis.

Once the half frequency $\sigma_{n k}$ of an inertial mode is found from (3.4), the explicit expression for the three velocity components for $\boldsymbol{u}_{n k}$ are

$$
\begin{aligned}
\hat{\boldsymbol{r}} \cdot \boldsymbol{u}_{n k}(r, \theta)= & -\mathrm{i} \sum_{i=0}^{k} \sum_{j=0}^{k-i} \frac{\mathscr{C}_{k i j}}{r}\left[\sigma_{n k}^{2}(i+j)-i\right] \\
& \times\left[r^{2(i+j)} \sigma_{n k}^{2 i-1}\left(1-\sigma_{n k}^{2}\right)^{j-1} \sin ^{2 j} \theta \cos ^{2 i} \theta\right], \\
\hat{\boldsymbol{\theta}} \cdot \boldsymbol{u}_{n k}(r, \theta)= & -\mathrm{i} \sum_{i=0}^{k} \sum_{j=0}^{k-i} \frac{\mathscr{C}_{k i j}}{r}\left[j \sigma_{n k}^{2} \cos ^{2} \theta+i\left(1-\sigma_{n k}^{2}\right) \sin ^{2} \theta\right] \\
& \times\left[r^{2(i+j)} \sigma_{n k}^{2 i-1}\left(1-\sigma_{n k}^{2}\right)^{j-1} \sin ^{2 j-1} \theta \cos ^{2 i-1} \theta\right], \\
\hat{\boldsymbol{\phi}} \cdot \boldsymbol{u}_{n k}(r, \theta)= & \sum_{i=0}^{k} \sum_{j=0}^{k-i} \frac{\mathscr{C}_{k i j}}{r} \sigma_{n k}^{2 i}\left(1-\sigma_{n k}^{2}\right)^{j-1} r^{2(i+j)} j \sin ^{2 j-1} \theta \cos ^{2 i} \theta,
\end{aligned}
$$

where $k \geqslant 2$ with $n=1,2, \ldots,(k-1)$, and the terms with $i+j=0$ in (3.6)-(3.8) should be excluded. Note that $\boldsymbol{u}_{n k}(r, \theta)$ satisfies the condition $\hat{\boldsymbol{r}} \cdot \boldsymbol{u}_{n k}=0$ at the bounding surface $\mathscr{S}$ of the spherical container. The analytical expression (3.6)-(3.8) represents all possible non-geostrophic inertial modes that may be excited by weak longitudinal libration.

Mathematically speaking, there exist the two conditions that must be satisfied in order that the Poincaré force, $\epsilon \hat{\omega} \cos (\hat{\omega} t) \boldsymbol{r} \times \hat{\boldsymbol{z}}$, can resonate with an inertial mode $\left(\omega_{n k}, \boldsymbol{u}_{n k}, p_{n k}\right)$ defined by (3.4) and (3.6)-(3.8). The first condition is that the libration frequency $\hat{\omega}$ must be equal or close to the frequency of the inertial mode, i.e. $\hat{\omega}=\omega_{n k}$. Obviously, this condition can be readily met by tuning the size of $\hat{\omega}$ in experiments or in any asymptotic or numerical analysis. In addition, the Poincare force, in order to resonate with the inertial mode $\left(\boldsymbol{u}_{n k}, p_{n k}, \omega_{n k}\right)$, must satisfies the second condition

$$
\int_{0}^{1} \int_{0}^{\pi} \int_{0}^{2 \pi} \boldsymbol{u}_{n k}^{*} \cdot[(\epsilon \hat{\omega}) \cos (\hat{\omega} t) \boldsymbol{r} \times \hat{z}] r^{2} \sin \theta \mathrm{d} \phi \mathrm{d} \theta \mathrm{d} r \neq 0
$$

where $\boldsymbol{u}_{n k}^{*}$ denotes the complex conjugate of $\boldsymbol{u}_{n k}$ defined by (3.6)-(3.8).

Unlike resonances in a precessing cylinder (for example, Gans 1970; Meunier et al. 2008), the second condition cannot be met in the present libration problem because

$$
\begin{aligned}
& \int_{0}^{1} \int_{0}^{\pi} \int_{0}^{2 \pi} \boldsymbol{u}_{n k}^{*} \cdot(\boldsymbol{r} \times \hat{\boldsymbol{z}}) r^{2} \sin \theta \mathrm{d} \phi \mathrm{d} \theta \mathrm{d} r \\
& =\frac{\mathrm{i}}{\omega_{n k}} \int_{0}^{1} \int_{0}^{\pi} \int_{0}^{2 \pi} \boldsymbol{u}_{n k}^{*} \cdot \nabla\left(|\boldsymbol{r} \times \hat{\boldsymbol{z}}|^{2}\right) r^{2} \sin \theta \mathrm{d} \phi \mathrm{d} \theta \mathrm{d} r \\
& =\frac{\mathrm{i}}{\omega_{n k}} \int_{0}^{\pi} \int_{0}^{2 \pi}\left[\left(\hat{\boldsymbol{r}} \cdot \boldsymbol{u}_{n k}^{*}\right)|\boldsymbol{r} \times \hat{\boldsymbol{z}}|^{2}\right]_{r=1} \sin \theta \mathrm{d} \phi \mathrm{d} \theta=0
\end{aligned}
$$

for all possible $n$ and $k$, where we have made use of $\boldsymbol{\nabla} \cdot \boldsymbol{u}_{n k}^{*}=0$ and the boundary condition $\hat{\boldsymbol{r}} \cdot \boldsymbol{u}_{n k}^{*}=0$ at $r=1$. Here $[f]_{r=x}$ denotes that the function $f$ evaluated at $r=x$. In deriving (3.10), we have made use of the inertial-mode equation satisfied by $\omega_{n k}$ and $\boldsymbol{u}_{n k}$. Note that a similar equation to (3.10) can be also derived for other axially symmetrical containers such as a longitudinally librating cylinder. 
According to our mathematical analysis, the Poincaré force due to spherical longitudinal libration is unable to resonate with any inertial mode $\boldsymbol{u}_{n k}$ given by (3.6)-(3.8) in a sphere. Physically, this non-resonant response stems from the spatiotemporal structure of the Poincaré force. All of the geostrophic modes are temporally non-oscillatory and, hence, cannot be resonantly excited by the oscillatory Poincaré force. All of the axisymmetric inertial modes are spatially non-geostrophic and, hence, cannot be resonantly excited by the spatially geostrophic Poincaré force. In contrast to the problem of a precessing cylinder in which $|\boldsymbol{u}|=O(\epsilon / \sqrt{E})$ at resonances, we would therefore expect that the amplitude of fluid motion in longitudinally librating spheres is always $|\boldsymbol{u}|=O(\epsilon)$ at $E \ll 1$ for any characteristic frequency $\omega_{n k}$.

\subsection{Asymptotic analysis at $\hat{\omega}=\omega_{n k}$}

Prior to presenting the detailed mathematical analysis, it is profitable to look at the physical picture of the problem. When the sphere is slightly librating with a moderate frequency $\hat{\omega} \gg E^{1 / 2}$, the resulting fluid motion far away from the spherical bounding surface $\mathscr{S}$ moves like an oscillatory solid-body rotation, $-\mathrm{i} \epsilon \boldsymbol{r} \times \hat{z} \mathrm{e}^{\mathrm{i} \hat{\omega} t}$, after the spin-up time scale $E^{-1 / 2}$. Since the container and the fluid is coupled purely by viscous processes, the fluid motion in the vicinity of $\mathscr{S}$ would vary rapidly and form a thin viscous boundary layer. The presence of the viscous boundary layer not only ensures that the interior oscillatory solid-body rotation satisfies the no-slip condition on $\mathscr{S}$ but also produces Ekman boundary pumping to drive the secondary oscillatory interior flow which would be dominated by a single inertial mode $\boldsymbol{u}_{n k}$ if $\hat{\omega}=\omega_{n k}$, as demonstrated by Aldridge \& Toomre (1969). Our analysis will concentrate on the three fundamental inertial modes with $\omega_{12}=1.309, \omega_{13}=0.9377$ and $\omega_{23}=1.6604$, even though the asymptotic solution would also be applicable to other higher modes.

It is the viscous boundary layer $\widetilde{\boldsymbol{u}}(r, \theta)$ that couples the oscillating solid-body rotation $-\mathrm{i} \epsilon \boldsymbol{r} \times \hat{\boldsymbol{z}} \mathrm{e}^{\mathrm{i} \hat{\omega} t}$ and the inertial mode $\boldsymbol{u}_{n k}$ together and, consequently, the three key elements of the solution should not be treated separately. This physical picture suggests the following asymptotic expansion for $E \ll 1$ at $\hat{\omega}=\omega_{n k}$ :

$$
\begin{aligned}
& \boldsymbol{u}(r, \theta, t)=\left[-\mathrm{i} \epsilon \boldsymbol{r} \times \hat{z}+\mathscr{A}_{n k} \boldsymbol{u}_{n k}(r, \theta)+\widehat{\boldsymbol{u}}(r, \theta)+\widetilde{\boldsymbol{u}}(r, \theta)\right] \mathrm{e}^{\mathrm{i} \hat{\omega} t}, \\
& p(r, \theta, t)=\left[\mathrm{i} \epsilon|\boldsymbol{r} \times \hat{z}|^{2}+\mathscr{A}_{n k} p_{n k}(r, \theta)+\widehat{p}(r, \theta)+\widetilde{p}(r, \theta)\right] \mathrm{e}^{\mathrm{i} \hat{\omega} t},
\end{aligned}
$$

where $\boldsymbol{u}_{n k}(r, \theta)$ and $p_{n k}(r, \theta)$ represents the axisymmetric inertial mode defined by (3.6)-(3.8) and (3.1), $\mathscr{A}_{n k}$ denotes the complex coefficient to be determined, and $\widehat{\boldsymbol{u}}$ and $\hat{p}$ are the interior perturbation produced primarily by the influx from the viscous boundary layer described by $\widetilde{\boldsymbol{u}}$ and $\widetilde{p}$. Note that the interior perturbation $\widehat{\boldsymbol{u}}(r, \theta)$ satisfies $|\widehat{\boldsymbol{u}}(r, \theta)| \ll\left|\mathscr{A}_{n k} \boldsymbol{u}_{n k}(r, \theta)\right|$ and, thus, can be neglected in the leading-order equation. We impose that

$$
\left(-\mathrm{i} \epsilon \boldsymbol{r} \times \hat{z}+\mathscr{A}_{n k} \boldsymbol{u}_{n k}+\widetilde{\boldsymbol{u}}\right)_{\mathscr{S}}=0
$$

on the bounding surface $\mathscr{S}$ such that the no-slip boundary condition is satisfied.

Inserting the interior part of the expansion (3.11)-(3.12) into (2.7)-(2.8) yields

$$
\begin{aligned}
\mathrm{i} \hat{\omega} \widehat{\boldsymbol{u}}+2 \hat{\boldsymbol{z}} \times \widehat{\boldsymbol{u}} & =-\nabla \widehat{p}, \\
\nabla \cdot \widehat{\boldsymbol{u}} & =0,
\end{aligned}
$$

subject to the boundary condition

$$
\hat{\boldsymbol{r}} \cdot \widehat{\boldsymbol{u}}=\sqrt{E} \int_{0}^{\infty} \hat{\boldsymbol{r}} \cdot[\nabla \times(\hat{\boldsymbol{r}} \times \widetilde{\boldsymbol{u}})] \mathrm{d} \xi,
$$


where $\xi=\sqrt{E}(1-r)$, the stretched boundary-layer coordinate for the boundary layer flow $\widetilde{\boldsymbol{u}}$. Homogeneous differential equations (3.14)-(3.15) together with the inhomogeneous boundary condition (3.16) requires the solvability condition,

$$
\int_{0}^{\pi}\left\{p_{n k}^{*}(r=1, \theta) \int_{0}^{\infty}\left[\frac{1}{\sin \theta} \frac{\partial}{\partial \theta} \sin \theta(\hat{\boldsymbol{\theta}} \cdot \widetilde{\boldsymbol{u}})\right] \mathrm{d} \xi\right\} \mathrm{d} \theta=0,
$$

which determines the amplitude $\mathscr{A}_{n k}$ for the inertial mode $\left(\boldsymbol{u}_{n k}, p_{n k}\right)$ forced by libration. It is essential that the solvability condition (3.17) is independent of the Ekman number $E$ and that the vanishing of the right-hand side of (3.17), in comparison to that of the precession problem, is equivalent to a physical statement for the absence of resonance.

The next major task is to derive the boundary flow $\widetilde{\boldsymbol{u}}$ needed in the solvability condition (3.17). By virtue of the properties of a viscous boundary layer in rapidly rotating spheres, we can derive a fourth-order differential equation from (2.7) governing the boundary-layer flow on $\mathscr{S}$,

$$
\left(\frac{\partial^{2}}{\partial \xi^{2}}-\mathrm{i} \hat{\omega}\right)^{2} \widetilde{\boldsymbol{u}}_{\text {tang }}+4(\hat{z} \cdot \hat{\boldsymbol{r}})^{2} \widetilde{\boldsymbol{u}}_{\text {tang }}=0,
$$

where $\widetilde{\boldsymbol{u}}_{\text {tang }}$ denotes the dominant tangential component of $\widetilde{\boldsymbol{u}}$, which can be readily solved subject to the following four conditions,

$$
\begin{aligned}
\left(\widetilde{\boldsymbol{u}}_{\text {tang }}\right)_{\xi=0} & =\left(\mathrm{i} \epsilon \boldsymbol{r} \times \hat{z}-\mathscr{A}_{n k} \boldsymbol{u}_{n k}\right)_{r=1}, \\
\left(\frac{\partial^{2} \widetilde{\boldsymbol{u}}_{\text {tang }}}{\partial \xi^{2}}\right)_{\xi=0} & =\mathrm{i} \hat{\omega}\left(\mathrm{i} \epsilon \boldsymbol{r} \times \hat{z}-\mathscr{A}_{n k} \boldsymbol{u}_{n k}\right)_{r=1}+2(\hat{z} \cdot \hat{\boldsymbol{r}}) \hat{\boldsymbol{r}} \times\left(\mathrm{i} \epsilon \boldsymbol{r} \times \hat{z}-\mathscr{A}_{n k} \boldsymbol{u}_{n k}\right)_{r=1}, \\
\left(\widetilde{\boldsymbol{u}}_{\text {tang }}\right)_{\xi \rightarrow \infty} & =0 \\
\left(\frac{\partial^{2} \widetilde{\boldsymbol{u}}_{\text {tang }}}{\partial \xi^{2}}\right)_{\xi \rightarrow \infty} & =0
\end{aligned}
$$

such that the no-slip condition (3.13) is explicitly enforced at the boundary $\mathscr{S}$. The fourth-order differential equation (3.18) together with the four boundary conditions determines the solution of the spherical Ekman boundary layer.

The solution to (3.18) that satisfies the four boundary conditions is

$$
\begin{aligned}
\widetilde{\boldsymbol{u}}_{\text {tang }}= & \frac{-1}{2}\left[\epsilon \sin \theta(\mathrm{i} \hat{\boldsymbol{\phi}}-\hat{\boldsymbol{\theta}})+\mathscr{A}_{n k}\left(\boldsymbol{u}_{n k}-\mathrm{i} \boldsymbol{r} \times \boldsymbol{u}_{n k}\right)_{r=1}\right] \mathrm{e}^{\gamma^{+} \xi} \\
& -\frac{1}{2}\left[\epsilon \sin \theta(\mathrm{i} \hat{\boldsymbol{\phi}}+\hat{\boldsymbol{\theta}})+\mathscr{A}_{n k}\left(\boldsymbol{u}_{n k}+\mathrm{i} \boldsymbol{r} \times \boldsymbol{u}_{n k}\right)_{r=1}\right] \mathrm{e}^{\gamma^{-\xi}},
\end{aligned}
$$

where

$$
\begin{aligned}
& \gamma^{+}=-\frac{\sqrt{2}}{2}\left[1+\frac{\mathrm{i}\left(\omega_{n k}+2 \cos \theta\right)}{\left|\omega_{n k}+2 \cos \theta\right|}\right]\left|\omega_{n k}+2 \cos \theta\right|^{1 / 2}, \\
& \gamma^{-}=-\frac{\sqrt{2}}{2}\left[1+\frac{\mathrm{i}\left(\omega_{n k}-2 \cos \theta\right)}{\left|\omega_{n k}-2 \cos \theta\right|}\right]\left|\omega_{n k}-2 \cos \theta\right|^{1 / 2},
\end{aligned}
$$

where $\mathscr{A}_{n k}$ is still unknown. In (3.20), there exist two critical colatitudes at which the boundary-layer solution breaks down as it thickens to $\mathrm{O}\left(E^{2 / 5}\right)$, as discussed by Roberts \& Stewartson (1965). However, it is generally believed that the effect of this type of singularities is not significant since the mass flux from the critical region is much smaller than that from the rest of the boundary layer (Roberts \& Stewartson 1965; Busse 1968; Hollerbach \& Kerswell 1995; Tilgner 1999). 
After making use of the boundary layer solution (3.20), the solvability condition (3.17) becomes

$$
\begin{aligned}
0= & \mathscr{A}_{n k} \int_{0}^{\pi} \frac{\partial p_{n k}^{*}(r=1, \theta)}{\partial \theta}\left[\hat{\boldsymbol{\theta}} \cdot \boldsymbol{u}_{n k}(r=1, \theta)+\mathrm{i} \hat{\boldsymbol{\phi}} \cdot \boldsymbol{u}_{n k}(r=1, \theta)\right] \\
& \times\left[\frac{1}{\left|\omega_{n k}+2 \cos \theta\right|^{1 / 2}}\left(1-\frac{\mathrm{i}\left(\omega_{n k}+2 \cos \theta\right)}{\left|\omega_{n k}+2 \cos \theta\right|}\right)\right] \sin \theta \mathrm{d} \theta \\
& +\mathscr{A}_{n k} \int_{0}^{\pi} \frac{\partial p_{n k}^{*}(r=1, \theta)}{\partial \theta}\left[\hat{\boldsymbol{\theta}} \cdot \boldsymbol{u}_{n k}(r=1, \theta)-\mathrm{i} \hat{\boldsymbol{\phi}} \cdot \boldsymbol{u}_{n k}(r=1, \theta)\right] \\
& \times\left[\frac{1}{\left|\omega_{n k}+2 \cos \theta\right|^{1 / 2}}\left(1-\frac{\mathrm{i}\left(\omega_{n k}-2 \cos \theta\right)}{\left|\omega_{n k}-2 \cos \theta\right|}\right)\right] \sin \theta \mathrm{d} \theta \\
& -\epsilon \int_{0}^{\pi} \frac{\partial p_{n k}^{*}(r=1, \theta)}{\partial \theta}\left[\frac{1}{\left|\omega_{n k}+2 \cos \theta\right|^{1 / 2}}\left(1-\frac{\mathrm{i}\left(\omega_{n k}+2 \cos \theta\right)}{\left|\omega_{n k}+2 \cos \theta\right|}\right)\right] \sin ^{2} \theta \mathrm{d} \theta \\
& \left.+\epsilon \int_{0}^{\pi} \frac{\partial p_{n k}^{*}(r=1, \theta)}{\partial \theta}\left[\frac{\mathrm{i}\left(\omega_{n k}-2 \cos \theta\right)}{\left|\omega_{n k}-2 \cos \theta\right|^{1 / 2}}\right)\right] \sin ^{2} \theta \mathrm{d} \theta
\end{aligned}
$$

This expression determines the amplitude $\mathscr{A}_{n k}$ of the inertial mode after evaluating several integrals there.

Two important features emerge from the solvability condition (3.22) without having to solve it. First, the Ekman number $E$ does not enter the solvability condition (3.22) in the present problem, a signature of the non-resonant response. This differs fundamentally from the resonant response that arises in problem of precession or latitudinal libration in an oblate spheroid (for example, Gans 1970; Zhang et al. 2012) in which the solvability condition explicitly depends on a $\sqrt{E}$ term. Second, there are no singular terms such as $1 /\left(\hat{\omega}-\omega_{n k}\right)$ in the solvability condition (3.22), which clearly indicates that the amplitude $\mathscr{A}_{n k}$ is proportional to $\epsilon$, another characteristic of the non-resonant response.

\subsection{Three fundamental modes}

There are three fundamental inertial modes, marked by the spatially simplest structure, in the subset of the inertial modes given by (3.6)-(3.8). The first fundamental inertial mode, whose pressure is a polynomial of $r$ of degree 4 , can be readily obtained by letting $k=2$ in (3.4) which yields

$$
\omega_{12}=2 \sigma_{12}=2 \sqrt{21} / 7 \approx 1.3093 .
$$

On inserting $\sigma_{12}=\sqrt{21} / 7$ into (3.1) and (3.6)-(3.8), we obtain the exact solution in closed form for the pressure $p_{12}$ and the velocity vector $\boldsymbol{u}_{12}$ in spherical polar coordinates

$$
\begin{aligned}
p_{12} & =\frac{15}{7}\left[-r^{2}\left(1+\frac{1}{2} \cos ^{2} \theta\right)+\frac{r^{4}}{4}\left(2+\cos ^{2} \theta+7 \sin ^{2} \theta \cos ^{2} \theta\right)\right], \\
\hat{\boldsymbol{r}} \cdot \boldsymbol{u}_{12} & =\frac{\mathrm{i} 15 \sqrt{3}}{8 \sqrt{7}}\left(r^{2}-1\right) r(1+3 \cos 2 \theta) \\
\hat{\boldsymbol{\theta}} \cdot \boldsymbol{u}_{12} & =\frac{\mathrm{i} 15 \sqrt{3}}{8 \sqrt{7}}\left(3-5 r^{2}\right) r \sin 2 \theta
\end{aligned}
$$




$$
\hat{\boldsymbol{\phi}} \cdot \boldsymbol{u}_{12}=\frac{15}{4}\left(-1+2 r^{2}+r^{2} \cos 2 \theta\right) r \sin \theta,
$$

representing the simplest solution of this subset.

On inserting the expressions of $\boldsymbol{u}_{12}, p_{12}^{*}$ and $\omega_{12}=2 \sqrt{21} / 7$ into the solvability condition (3.22) and carrying out the relevant integrals, we obtain that

$$
\mathscr{A}_{12}=(0.03416-\mathrm{i} 0.1364) \epsilon .
$$

It follows that the asymptotic solution for the pressure $p$ at $E \ll 1$ is

$$
\begin{aligned}
p(r, \theta, t)= & \epsilon r^{2}\left\{\operatorname{isin}^{2} \theta+\frac{15(0.03416-\mathrm{i} 0.1364)}{7}\right. \\
& \left.\times\left[-\left(1+\frac{1}{2} \cos ^{2} \theta\right)+\frac{r^{2}}{4}\left(2+\cos ^{2} \theta+7 \sin ^{2} \theta \cos ^{2} \theta\right)\right]\right\} \mathrm{e}^{\mathrm{i} 2 \sqrt{21} t / 7},
\end{aligned}
$$

while its corresponding velocity $\boldsymbol{u}$ satisfying the no-slip condition is

$$
\begin{aligned}
\boldsymbol{u}(r, \theta, t)= & \epsilon\left\{r \sin \theta\left[\mathrm{i} r \hat{\boldsymbol{\phi}}-\frac{1}{2}(\mathrm{i} \hat{\boldsymbol{\phi}}-\hat{\boldsymbol{\theta}}) \mathrm{e}^{\gamma_{21}^{+} \xi}-\frac{1}{2}(\mathrm{i} \hat{\boldsymbol{\phi}}+\hat{\boldsymbol{\theta}}) \mathrm{e}^{\gamma_{21}^{-} \xi}\right]\right. \\
& +\frac{15(0.034156-\mathrm{i} 0.13641)}{4}\left[\frac{\mathrm{i} \sqrt{3}}{2 \sqrt{7}}\left(r^{3}-r\right)(1+3 \cos 2 \theta) \hat{\boldsymbol{r}}\right. \\
& +\frac{\mathrm{i} \sqrt{3}}{2 \sqrt{7}} \sin 2 \theta\left(3 r-5 r^{3}\right) \hat{\boldsymbol{\theta}}+\sin \theta\left(2 r^{3}+r^{3} \cos 2 \theta-r\right) \hat{\boldsymbol{\phi}} \\
& +\frac{\mathrm{i}}{2}\left(1+\cos 2 \theta-\frac{\sqrt{12}}{\sqrt{7}} \cos \theta\right) \sin \theta(\mathrm{i} \hat{\boldsymbol{\phi}}-\hat{\boldsymbol{\theta}}) \mathrm{e}^{\gamma_{21}^{+} \xi} \\
& \left.\left.+\frac{\mathrm{i}}{2}\left(1+\cos 2 \theta+\frac{\sqrt{12}}{\sqrt{7}} \cos \theta\right) \sin \theta(\mathrm{i} \hat{\boldsymbol{\phi}}+\hat{\boldsymbol{\theta}}) \mathrm{e}^{\gamma_{21}^{-} \xi}\right]\right\} \mathrm{e}^{\mathrm{i} 2 \sqrt{21} t / 7},
\end{aligned}
$$

where

$$
\begin{aligned}
& \gamma_{21}^{+}=-\left[1+\frac{\mathrm{i}(\sqrt{21} / 7+\cos \theta)}{|\sqrt{21} / 7+\cos \theta|}\right]|\sqrt{21} / 7+\cos \theta|^{1 / 2}, \\
& \gamma_{21}^{-}=-\left[1+\frac{\mathrm{i}(\sqrt{21} / 7-\cos \theta)}{|\sqrt{21} / 7-\cos \theta|}\right]|\sqrt{21} / 7-\cos \theta|^{1 / 2} .
\end{aligned}
$$

Note that the Ekman number $E$ does not appear either in the pressure expression (3.29) or the amplitude of the velocity (3.30), both of which are only valid for $E \ll 1$. The spatial structure of the librating flow $\boldsymbol{u}$ for $\epsilon=0.01$ and $\hat{\omega}=\omega_{12}=1.309$, computed from the expression (3.30) but excluding the boundary-layer term, is depicted in figure $1(a, b)$, which will be compared with the corresponding numerical solutions. Since the flow is oscillatory, we have chosen to plot it at the instant when its kinetic energy reaches the maximum: this instant is identified in both linear and nonlinear numerical solutions.

For illuminating the non-resonant feature of the problem, we introduce an average kinetic energy density $\bar{E}_{k i n}$, a quantity measuring the flow amplitude $|\boldsymbol{u}|$ and being 
(a)

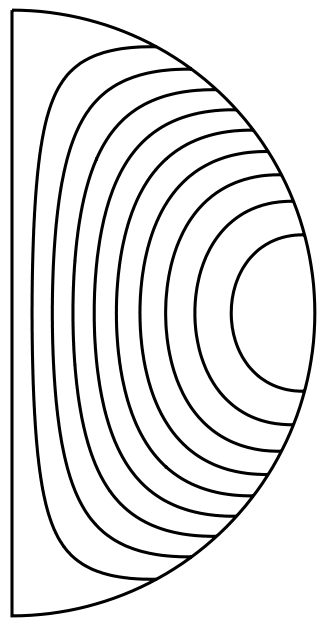

(c)

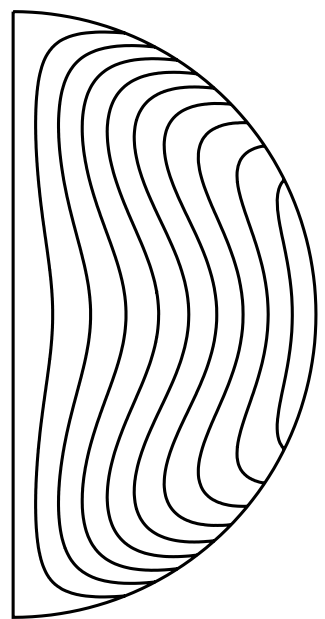

(b)

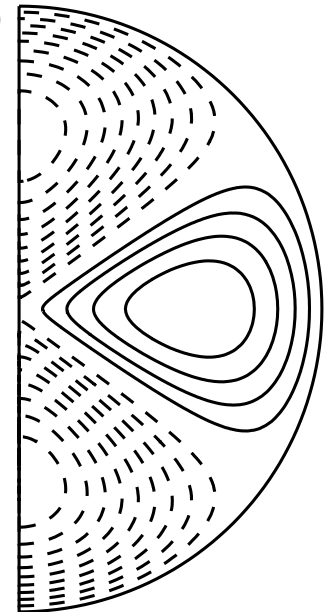

(d)

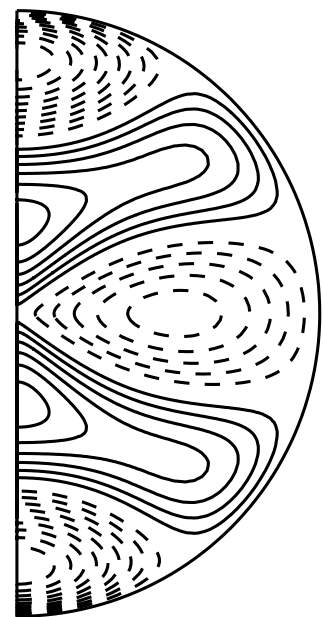

FIGURE 1. (a) Contours of the azimuthal component $\hat{\boldsymbol{\phi}} \cdot \boldsymbol{u}$ and $(b)$ contours of the radial component $\hat{\boldsymbol{r}} \cdot \boldsymbol{u}$ for $\hat{\omega}=1.3093$ computed from the analytical expression (3.30) in a meridional plane. Note that the boundary-layer term in (3.30) is not included in the plot $\hat{\boldsymbol{\phi}} \cdot \boldsymbol{u}$. (c) Contours of $\hat{\boldsymbol{\phi}} \cdot \boldsymbol{u}$ and $(d)$ contours of $\hat{\boldsymbol{r}} \cdot \boldsymbol{u}$ for $\hat{\omega}=1.6604$ computed from the analytical expression. The dashed contours are for $\hat{\boldsymbol{r}} \cdot \boldsymbol{u}<0$ (or $\hat{\boldsymbol{\phi}} \cdot \boldsymbol{u}<0$ ) while the solid contours are for $\hat{\boldsymbol{r}} \cdot \boldsymbol{u}>0$ ( or $\hat{\boldsymbol{\phi}} \cdot \boldsymbol{u}>0$ ). All of the solutions are plotted at the instant when the kinetic energy reaches its maximum for $\epsilon=0.01$.

easily comparable to the numerical solutions, defined as

$$
\bar{E}_{k i n}=\left(\frac{1}{2 V}\right)\left(\frac{1}{2 \pi / \hat{\omega}}\right) \int_{0}^{2 \pi / \hat{\omega}}\left(\int_{0}^{1} \int_{0}^{2 \pi} \int_{0}^{\pi}|\operatorname{Real}\{\boldsymbol{u}\}|^{2} r^{2} \sin \theta \mathrm{d} \theta \mathrm{d} \phi \mathrm{d} r\right) \mathrm{d} t,
$$

where $V$ is the volume of the sphere $V=4 \pi / 3$. The small contribution to $\bar{E}_{k i n}$ from the viscous boundary layer is neglected, implying that the kinetic energy $\bar{E}_{k i n}$ would be 
slightly overestimated. Making use of the expression (3.30), we obtain that

$$
\begin{aligned}
\bar{E}_{k i n} & =\frac{\epsilon^{2}}{10}+\frac{3}{16 \pi}\left|\mathscr{A}_{12}\right|^{2} \int_{0}^{1} \int_{0}^{2 \pi} \int_{0}^{\pi}\left|\boldsymbol{u}_{12}\right|^{2} r^{2} \sin \theta \mathrm{d} \theta \mathrm{d} \phi \mathrm{d} r \\
& =\left[\frac{1}{10}+0.0106\right] \epsilon^{2}
\end{aligned}
$$

for the first fundamental mode. The first term in the square brackets represents the contribution from inviscid Poincaré flow in the bulk fluid. The second term represents the contribution arising from any viscously excited inertial modes.

Two other fundamental modes in the subset have the pressure of a polynomial $r$ of degree 6 corresponding to $k=3$ in (3.1) with its half-frequency being solutions to

$$
33 \sigma_{n 3}^{4}-30 \sigma_{n 3}^{2}+5=0
$$

which has two positive roots

$$
\begin{aligned}
& \omega_{13}=2 \sigma_{13}=2 \sqrt{\frac{15-\sqrt{60}}{33}} \approx 0.9377, \\
& \omega_{23}=2 \sigma_{23}=2 \sqrt{\frac{15+\sqrt{60}}{33}} \approx 1.6604 .
\end{aligned}
$$

Computed using the solvability condition (3.22) with $\omega_{13}=2[(15-\sqrt{60}) / 33]^{1 / 2}$, we obtain

$$
\mathscr{A}_{13}=(-0.0084+\mathrm{i} 0.0700) \epsilon \text {. }
$$

The leading-order velocity $\boldsymbol{u}$ satisfying the no-slip condition, the pressure $p$ and the average kinetic energy $\bar{E}_{k i n}$ at the libration frequency $\hat{\omega}=0.9377$ are then

$$
\begin{aligned}
\boldsymbol{u}(r, \theta, t) & =\left[-\mathrm{i} \epsilon \boldsymbol{r} \times \hat{z}+\epsilon(-0.0084+\mathrm{i} 0.0700) \boldsymbol{u}_{13}+\widetilde{\boldsymbol{u}}_{t a n g}\right] \mathrm{e}^{\mathrm{i} 0.9377 t}, \\
p(r, \theta, t) & =\epsilon\left[\mathrm{i}|\boldsymbol{r} \times \hat{z}|^{2}+(-0.0084+\mathrm{i} 0.0700) p_{13}\right] \mathrm{e}^{\mathrm{i} 0.9377 t}, \\
\bar{E}_{k i n} & =\left[\frac{1}{10}+0.0068\right] \epsilon^{2},
\end{aligned}
$$

where $\left(\boldsymbol{u}_{13}, p_{13}\right)$ is the exact eigenfunction in closed form given by (3.6)-(3.8) and (3.1) while $\widetilde{\boldsymbol{u}}_{\text {tang }}$ given by (3.20) together with $\omega_{13}=2[(15-\sqrt{60}) / 33]^{1 / 2}$.

Similarly, the solvability condition (3.22) with $\omega_{23}=2 \sigma_{23}=2[(15+\sqrt{60}) / 33]^{1 / 2}=$ 1.6604 , after carrying out the four integrals, gives

$$
\mathscr{A}_{23}=(-0.0057+\mathrm{i} 0.0183) \epsilon \text {. }
$$

The leading-order velocity satisfying the no-slip condition, the pressure and the average kinetic energy when $\hat{\omega}=1.6605$ are then

$$
\begin{aligned}
\boldsymbol{u}(r, \theta, t) & =\left[-\mathrm{i} \epsilon \boldsymbol{r} \times \hat{z}+\epsilon(-0.0057+\mathrm{i} 0.0183) \boldsymbol{u}_{23}+\widetilde{\boldsymbol{u}}_{\text {tang }}\right] \mathrm{e}^{\mathrm{i} 1.6604 t}, \\
p(r, \theta, t) & =\epsilon\left[\mathrm{i}|\boldsymbol{r} \times \hat{z}|^{2}+(-0.0057+\mathrm{i} 0.0183) p_{23}\right] \mathrm{e}^{\mathrm{i} 1.6604 t}, \\
\bar{E}_{k i n} & =\left[\frac{1}{10}+0.0019\right] \epsilon^{2},
\end{aligned}
$$

where $\left(\boldsymbol{u}_{23}, p_{23}\right)$ is the exact eigenfunction in closed form given by (3.6)-(3.8) and (3.1) while $\widetilde{\boldsymbol{u}}_{\text {tang }}$ given by (3.20) with $\omega_{23}=2[(15+\sqrt{60}) / 33]^{1 / 2}$. The spatial structure 
of the flow $\boldsymbol{u}$ for $\hat{\omega}=1.6604$ and $\epsilon=0.01$, valid for any $E \ll 1$ and computed from the above analytical expression, is depicted in figure $1(c, d)$ at the instant when its kinetic energy reaches the maximum.

\subsection{Structure of the asymptotic solution}

An advantage of having an explicitly analytical solution for both $\boldsymbol{u}$ and $\boldsymbol{p}$ that satisfies all of the required boundary condition, such as (3.29) and (3.30), is to provide a theoretical basis by which to interpret complicated experimental or numerical results. As clearly indicated in the pressure expressions of the three fundamental modes, the contribution from the second term whose amplitude depends on the libration frequency $\hat{\omega}$ is, in general, always small compared with the first dominant term, representing the inviscid response to libration, whose amplitude is independent of $\hat{\omega}$. The dominant first term, however, always vanishes on the rotation axis $s=0$. In consequence, the pressure difference measured on the rotation axis $s=0$ is strongly dependent on $\hat{\omega}$, whilst it is nearly constant when evaluated at other locations, such as on the equatorial plane $z=0$ where the first term is dominant. In other words, the theory suggests that the maximum pressure differences measured in the equatorial plane, for example between the equator and the centre, would always be given by

$$
\frac{1}{\epsilon}\left|p\left(\theta=\frac{\pi}{2}, r=1, t\right)-p\left(\theta=\frac{\pi}{2}, r=0, t\right)\right|_{\max }=1+\text { small perturbations }
$$

for any values of $0<\hat{\omega}<2$ at $E \ll 1$. Similarly, the analytical expressions for $\boldsymbol{u}$ also suggest that the contribution from the second term whose amplitude would depend on $\hat{\omega}$ is always small compared with the first term whose amplitude is independent of $\hat{\omega}$. The analytical solutions suggest that the scaled average kinetic energy $\bar{E}_{k i n} / \epsilon^{2}$ of the librating flow would be always given by

$$
\frac{\bar{E}_{k i n}}{\epsilon^{2}}=\frac{1}{10}+\text { small perturbations }
$$

for any values of $0<\hat{\omega}<2$ at $E \ll 1$. Those theoretical predictions will be confirmed by both linear and nonlinear numerical analysis.

\section{Linear numerical analysis}

The primary purpose of our linear numerical analysis, which is valid for both large and small Ekman numbers but cannot produce the pressure field directly, is to validate the asymptotic solution that is valid only for $E \ll 1$ as well as to compare with the nonlinear solution at $\epsilon \ll 1$.

In the numerical analysis, we expand the velocity $\boldsymbol{u}$ as a sum of poloidal $(v)$ and toroidal $(w)$ components by writing

$$
\boldsymbol{u}(r, \theta, t)=\{\nabla \times \nabla \times[\boldsymbol{r} v(r, \theta)]+\nabla \times[\boldsymbol{r} w(r, \theta)]\} \mathrm{e}^{\mathrm{i} \hat{\omega} t},
$$

where $\boldsymbol{r}$ is the position vector. Obviously, the condition $\boldsymbol{\nabla} \cdot \boldsymbol{u}=0$ is automatically satisfied. Inserting (4.1) into (2.7) and, then, applying $\boldsymbol{r} \cdot \boldsymbol{\nabla}$ and $\boldsymbol{r} \cdot \boldsymbol{\nabla} \times \boldsymbol{\nabla}$ onto the resulting equation, we derive the two independent scalar equations,

$$
\begin{gathered}
\left(\mathrm{i} \hat{\omega}-E \Delta^{2}\right)\left(\frac{1}{\sin \theta} \frac{\partial}{\partial \theta} \sin \theta \frac{\partial}{\partial \theta}\right) \Delta^{2} v+2 \mathscr{Q}_{2} w=0, \\
\left(\mathrm{i} \hat{\omega}-E \Delta^{2}\right)\left(\frac{1}{\sin \theta} \frac{\partial}{\partial \theta} \sin \theta \frac{\partial}{\partial \theta}\right) w-2 \mathscr{Q}_{2} v=2 \epsilon \hat{\omega} r \cos \theta
\end{gathered}
$$


where the differential operator $\Delta^{2}$ is

$$
\Delta^{2}=\frac{1}{r^{2}} \frac{\partial}{\partial r} r^{2} \frac{\partial}{\partial r}+\frac{1}{r^{2} \sin \theta} \frac{\partial}{\partial \theta} \sin \theta \frac{\partial}{\partial \theta},
$$

and the differential operator $\mathscr{Q}_{2}$ is connected with the effects of rotation and is defined as

$$
\mathscr{Q}_{2}=r \cos \theta \Delta^{2}+\left(\frac{1}{\sin \theta} \frac{\partial}{\partial \theta} \sin \theta \frac{\partial}{\partial \theta}-r \frac{\partial}{\partial r}\right)\left(\cos \theta \frac{\partial}{\partial r}-\frac{\sin \theta}{r} \frac{\partial}{\partial \theta}\right) .
$$

In terms of $v$ and $w$, the non-slip boundary condition becomes

$$
v=\frac{\partial v}{\partial r}=w=0 \quad \text { at } r=1 .
$$

Equations (4.2)-(4.3) subject to the condition (4.6) are solved numerically by expanding the velocity potentials $v$ and $w$ in terms of Legendre function $P_{l}(\cos \theta)$ and of the radial functions that satisfy both the central condition at $r=0$ and the no-slip condition $r=1$, which are

$$
\begin{aligned}
w(r, \theta) & =\sum_{l=1}^{L} \sum_{n=0}^{N} w_{l n} r^{l}(1-r) T_{n}(2 r-1) P_{2 l-1}(\cos \theta), \\
v(r, \theta) & =\sum_{l=1}^{L} \sum_{n=0}^{N} v_{l n} r^{l}(1-r)^{2} T_{n}(2 r-1) P_{2 l}(\cos \theta),
\end{aligned}
$$

where $T_{n}(x)$ denotes the standard Chebyshev function, $w_{l n}$ and $v_{l n}$ are complex coefficients to be determined and $N$ and $L$ are truncation parameters whose size is determined by the size of $E$ (for $E=10^{-4}$ we usually take $N=L=50$ for better than $1 \%$ accuracy). The Legendre function $P_{l}(\cos \theta)$ is normalized such that

$$
\frac{1}{2} \int_{0}^{\pi}\left|P_{l}(\cos \theta)\right|^{2} \sin \theta \mathrm{d} \theta=1 .
$$

It should be noted that the selection of $l$ in $P_{l}(\cos \theta)$ for the above expansion ensures the equatorial symmetry of the librating flow obeying the parity

$$
w(r, \theta)=-w(r, \pi-\theta) \text { and } v(r, \theta)=v(r, \pi-\theta),
$$

while the factor $r^{l}$ in the expansions is required to remove the singularity at the origin $r=0$. On substitution of the expansions into (4.2)-(4.3) along with an application of the standard numerical procedure, we are able to derive a system of linear algebraic equations for the coefficients $w_{l n}$ and $v_{l n}$ which are then solved numerically by an iterative method.

It is found that, when $E \leqslant 10^{-4}$, the linear numerical solutions approach the asymptotic regime such that they become comparable to the asymptotic solution valid only for $E \ll 1$. In order to compare directly with both the analytical expression and the fully nonlinear numerical solution, figure 2 shows the spatial structures of $\boldsymbol{u}$ for $\hat{\omega}=1.3093$ (figure $2 a, b$ ) and $\hat{\omega}=1.6604$ (figure $2 c, d$ ) at exactly the same parameters as those in figure 1. More discussions about the linear numerical solution are presented in $\S 7$. 
(a)

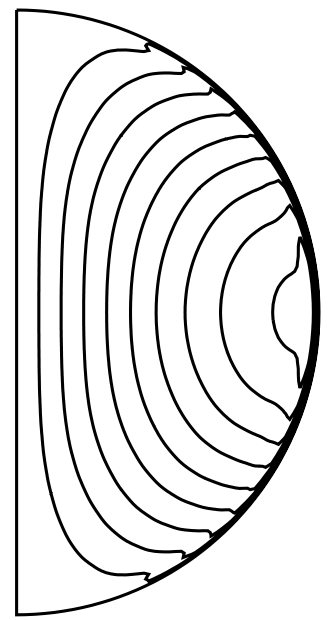

(c)

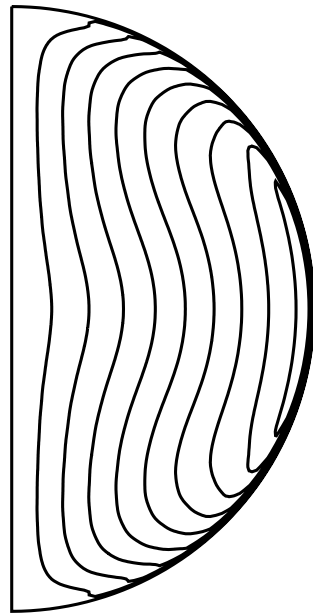

(b)

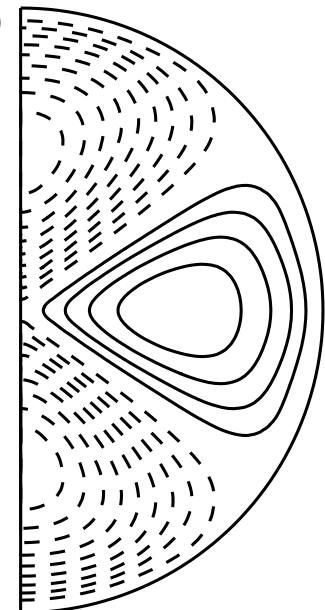

(d)

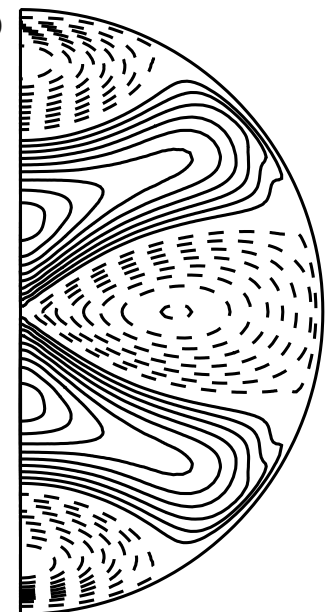

FIGURE 2. (a) Contours of the azimuthal component $\hat{\boldsymbol{\phi}} \cdot \boldsymbol{u}$ and $(b)$ contours of the radial component $\hat{\boldsymbol{r}} \cdot \boldsymbol{u}$ for $\hat{\omega}=1.3093$ computed from the linear numerical solution. (c) Contours of $\hat{\boldsymbol{\phi}} \cdot \boldsymbol{u}$ and $(d)$ contours of $\hat{\boldsymbol{r}} \cdot \boldsymbol{u}$ for $\hat{\omega}=1.6604$ computed from the linear numerical solution in a meridional plane. All of the solutions are plotted at the instant when the kinetic energy reaches its maximum $E=10^{-4}$ and $\epsilon=0.01$.

\section{Nonlinear numerical simulation}

We employ a finite-element method based on a three-dimensional tetrahedralization of the whole sphere with a uniform mesh distribution on a spherical surface without having the pole singularity and a small number of nodes with a nearly uniform mesh distribution in the neighbourhood of the centre $r=0$ without having the central singularity. Moreover, the three-dimensional mesh is constructed in a way such that more nodes placed in the vicinity of the bounding surface for the purpose of resolving the spherical viscous boundary layer. The detail of the numerical method and its validation can be found in Chan, Zhang \& Liao (2010). An advantage of using a finite-element method in this particular problem is that the pressure $p$, in addition to the velocity $\boldsymbol{u}$, are obtained directly from numerical simulation. Of course, in principle, 
we can find the pressure $p$ in the linear analysis after obtaining $v$ and $w$ by solving the equation

$$
2 \nabla \cdot\{\hat{z} \times[\nabla \times \nabla \times(\boldsymbol{r} v(r, \theta))+\nabla \times(\boldsymbol{r} w(r, \theta))]\}=-\nabla^{2} p .
$$

Evidently, solving the above equation with a complicated $v$ and $w$ is certainly not straightforward. Moreover, since all of the terms in (2.3) are retained in the nonlinear problem with no spatial symmetries being imposed, the nonlinear solutions not only enables us to validate the linear approximation in the asymptotic analysis for $\epsilon \ll 1$ but also would reveal the existence of other possible resonances that are only connected with the nonlinear effects (see, for example, Noir et al. 2003).

Our finite-element code makes use of a semi-implicit time-stepping scheme with an implicit second-order backward differentiation formula and a second-order extrapolation for the nonlinear term. With this numerical scheme, the fully nonlinear equations (2.3)-(2.4) are written in the form

$$
\begin{gathered}
\frac{3 \boldsymbol{u}^{n+1}-4 \boldsymbol{u}^{n}+\boldsymbol{u}^{n-1}}{2 \Delta t}+2\left(\boldsymbol{u}^{n} \cdot \nabla \boldsymbol{u}^{n}\right)-\left(\boldsymbol{u}^{n-1} \cdot \nabla \boldsymbol{u}^{n-1}\right)+2\left[1+\epsilon \sin \left(\hat{\omega} t_{n+1}\right)\right] \hat{z} \times \boldsymbol{u}^{n+1} \\
=-\nabla p^{n+1}+E \nabla^{2} \boldsymbol{u}^{n+1}+(\hat{\omega} \epsilon) \boldsymbol{r} \times \hat{z} \cos \left(\hat{\omega} t_{n+1}\right) \\
\boldsymbol{\nabla} \cdot \boldsymbol{u}^{n+1}=0
\end{gathered}
$$

where $t_{n}=n \Delta t$ for $n=0,1, \ldots$ with $\boldsymbol{u}^{n}(\boldsymbol{r})=\boldsymbol{u}\left(\boldsymbol{r}, t_{n}\right)$. They are solved, starting from an arbitrary initial condition, to find $\boldsymbol{u}^{n+1}, p^{n+1}$ for given $\boldsymbol{u}^{n}$ and $p^{n}$ on modern parallel computers. It is also noteworthy that, because the coupling between the oscillating container and the fluid is purely viscous, the three-dimensional simulation is computationally expensive: it usually takes more than $O\left(E^{-1 / 2}\right)$ dimensionless time units to reach a nonlinear equilibrium from an arbitrary initial state for $E \ll 1$. Our typical nonlinear simulation uses a spherical finite-element mesh with 302988 tetrahedral elements and a total of 1152054 unknowns.

The structure of the nonlinear librating flow $\boldsymbol{u}$ for $\hat{\omega}=1.3093$ and $\hat{\omega}=1.6604$ simulated at $E=10^{-4}$ and $\epsilon=0.01$ is depicted in figure 3, which has the same parameters as those used in figure 1 for the analytical solution and in figure 2 for the linear numerical solution. The results of our nonlinear numerical simulation supports that: (i) the linear approximation is appropriate for $\epsilon \ll 1$ at $E \ll 1$; and (ii) no other types of resonances related to nonlinear effects exist when $\epsilon \ll 1$. More discussions about the nonlinear three-dimensional simulations are presented in $\S 7$.

\section{Comparison: asymptotic versus numerical solutions}

To answer the two questions raised in $\S 1$, it suffices to examine the dynamical responses of the librating sphere in the neighbourhood of the first fundamental inertial mode with $\omega_{12}=1.3093$. An extensive numerical analysis is carried out in the neighbourhood of the libration frequency $\hat{\omega}=1.3093$ for fixed $E=10^{-4}$ and $\epsilon=0.01$. For an easy comparison, the results obtained using the three different methods at the same parameter are summarized in figure 4.

The solid curve labelled by $A$ in figure 4 shows $\log _{10}|\Delta p(\theta=\pi / 2) / \epsilon|$, where

$$
\Delta p\left(\theta=\frac{\pi}{2}\right)=\left|p\left(\theta=\frac{\pi}{2}, r=1, t\right)-p\left(\theta=\frac{\pi}{2}, r=\frac{1}{2}, t\right)\right|_{\max }
$$

represents the maximum pressure difference on the equatorial plane between $(\theta=$ $\pi / 2, r=1)$ and $(\theta=\pi / 2, r=1 / 2)$, as a function of the libration frequency $\hat{\omega}$. 
(a)

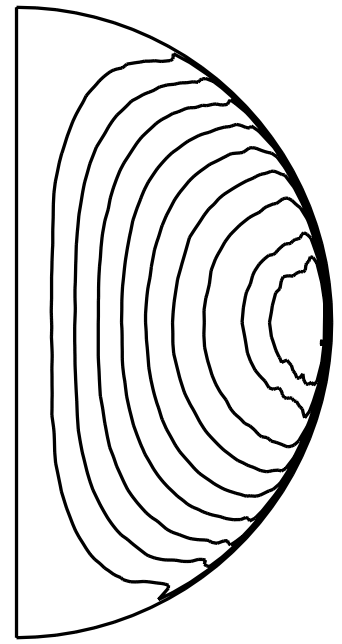

(c)

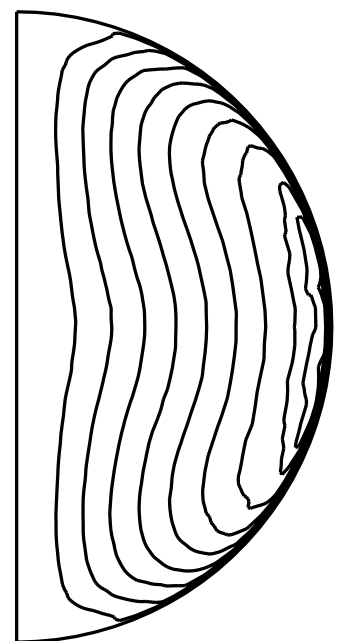

(b)

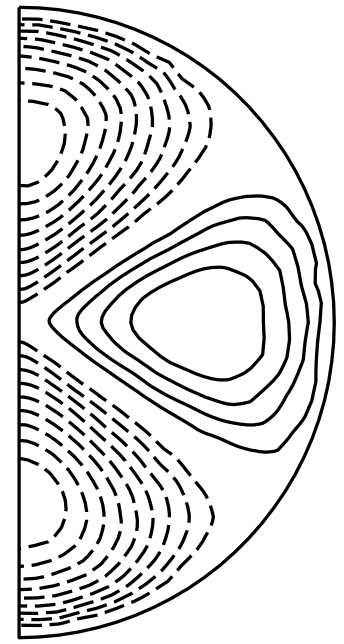

(d)

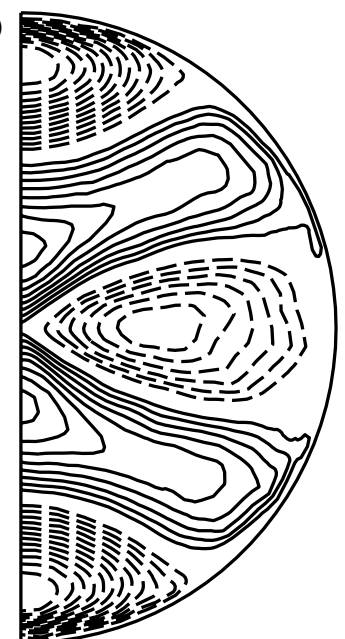

Figure 3. (a) Contours of the azimuthal component $\hat{\boldsymbol{\phi}} \cdot \boldsymbol{u}$ and $(b)$ the radial component $\hat{\boldsymbol{r}} \cdot \boldsymbol{u}$ for $\hat{\omega}=1.3093$ computed from nonlinear numerical solution. (c) Contours of $\hat{\boldsymbol{\phi}} \cdot \boldsymbol{u}$ and $(d) \hat{\boldsymbol{r}} \cdot \boldsymbol{u}$ for $\hat{\omega}=1.6604$ computed from nonlinear numerical solution. All of the solutions are plotted at the instant when the kinetic energy reaches its maximum $E=10^{-4}$ and $\epsilon=0.01$.

There is a square on the curve $A$ in figure 4 representing the value computed from the analytical solution (3.29). The solid curve labelled by $C$ in figure 4 shows $\log _{10}|3 \Delta p(\theta=0) / \epsilon|$, where

$$
\Delta p(\theta=0)=\left|p(\theta=0, r=1, t)-p\left(\theta=0, r=\frac{1}{2}, t\right)\right|_{\max }
$$

represents the maximum pressure difference on the rotation axis between $(\theta=0, r=$ $1)$ and $(\theta=0, r=1 / 2)$, as a function of the libration frequency $\hat{\omega}$. A factor of three is included in order to fit the curve into the figure. The detail of the nonlinear numerical solution used for plotting figure 4 is given in table 1 .

Our results reveal that the pressure difference on the rotation axis $\Delta p(\theta=0)$ reaches a sharp peak (noting the curve in figure 4 takes the logarithm scaling) when $\hat{\omega}$ 


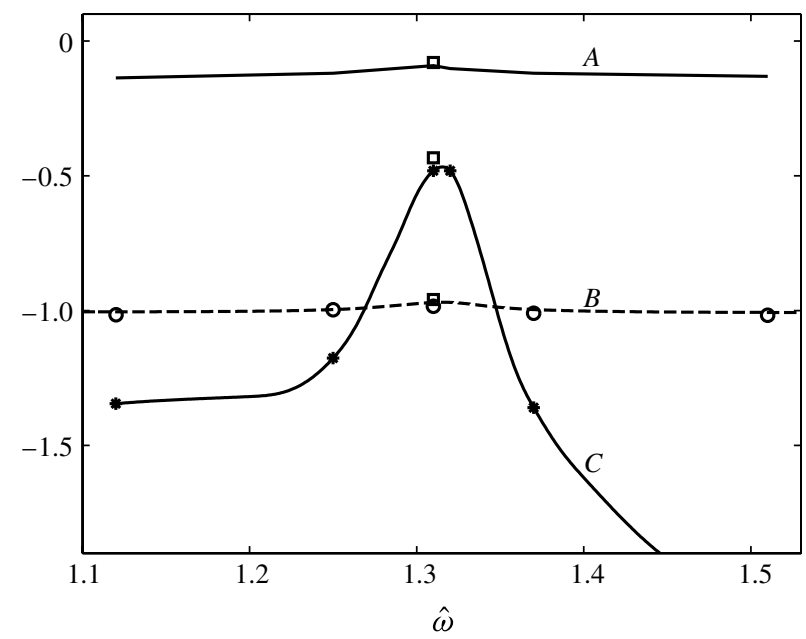

FIgURE 4. The solid curve labelled by $A$ represents $\log |\Delta p(\theta=\pi / 2) / \epsilon|$ as a function of $\hat{\omega}$ on the equatorial plane from the result of nonlinear numerical simulation, with the square on the curve being from the analytical solution. The dashed curve labelled by $B$ represents the scaled kinetic energy $\log \left(\bar{E}_{k i n} / \epsilon^{2}\right)$ computed from the linear numerical analysis as a function of the libration frequency $\hat{\omega}$. On the dashed curve, the square is calculated from the analytical solution while the circles are calculated from the result of nonlinear numerical simulation. The solid curve labelled by $C$ represents $\log |3 \Delta p(\theta=0) / \epsilon|$ as a function of $\hat{\omega}$ along the rotation axis from the result of nonlinear numerical simulation, with the square being computed from the analytical solution and the asterisks showing five solutions of the nonlinear simulation given in table 1 . The parameters for all of the solutions are $E=10^{-4}$ and $\epsilon=0.01$ with the pressure difference being evaluated at the instant when its kinetic energies reach the maximum.

$\begin{array}{lccc}\hat{\omega} & |\Delta p(\theta=0) / \epsilon| & |\Delta p(\theta=\pi / 2) / \epsilon| & \bar{E}_{k i n} / \epsilon^{2} \\ 0.9377 & 9.881 \times 10^{-2} & 7.765 \times 10^{-1} & 9.799 \times 10^{-2} \\ 1.1235 & 1.505 \times 10^{-2} & 7.257 \times 10^{-1} & 9.641 \times 10^{-2} \\ 1.2500 & 2.223 \times 10^{-2} & 7.604 \times 10^{-1} & 1.007 \times 10^{-1} \\ 1.3093 & 1.075 \times 10^{-1} & 8.065 \times 10^{-1} & 1.036 \times 10^{-1} \\ 1.3190 & 1.134 \times 10^{-1} & 7.905 \times 10^{-1} & 1.019 \times 10^{-1} \\ 1.3700 & 1.456 \times 10^{-2} & 7.565 \times 10^{-1} & 9.776 \times 10^{-2} \\ 1.5093 & 3.180 \times 10^{-3} & 7.444 \times 10^{-1} & 9.616 \times 10^{-2} \\ 1.6604 & 2.638 \times 10^{-2} & 7.214 \times 10^{-1} & 9.637 \times 10^{-2}\end{array}$

TABLE 1. Several results of the fully nonlinear numerical simulation for $E=10^{-4}$ and $\epsilon=0.01$ in a librating fluid sphere.

approaches the characteristic value of the first fundamental mode $\omega_{12}=1.3093$, which is consistent with the experimental observation (figures 3 and 4 of Aldridge \& Toomre 1969). However, the pressure difference on the equatorial plane $\Delta p(\theta=\pi / 2)$, which is always much larger than that on the rotation axis, i.e.

$$
\left|p\left(\frac{\pi}{2}, 1, t\right)-p\left(\frac{\pi}{2}, \frac{1}{2}, t\right)\right|_{\max } \gg\left|p(0,1, t)-p\left(0, \frac{1}{2}, t\right)\right|_{\max },
$$




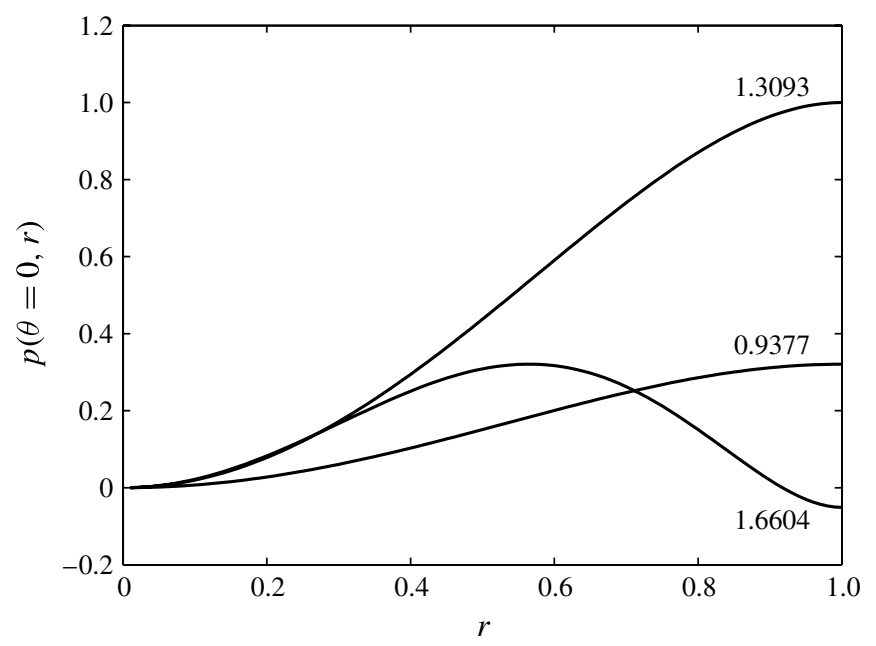

FIgURE 5. Pressure along the rotation axis, $p(\theta=0, r)$, plotted according to the expression (3.1), for the three fundamental modes $\omega_{12}=1.309, \omega_{13}=0.9377$ and $\omega_{23}=1.6604$.

is nearly independent of $\hat{\omega}$ at $E \ll 1$ and can always be expressed as 1 minus some perturbations. This property can be readily explained, as discussed previously, by looking at the structure of the analytical expressions. It is important to note that the particular structure of the pressure field given by (3.29) possesses a remarkable property: measuring the pressure difference $\Delta p(\theta=0)$ on the rotation axis picks up weaker signals of the small secondary flow in the form of an oscillatory inertial mode because the dominant, inviscid, solid body term vanishes on the axis.

The dashed curve labelled by $B$ in figure 4 shows $\log _{10}\left(\bar{E}_{k i n} / \epsilon^{2}\right)$, the scaled kinetic energies of the librating flow, computed from three different methods, as a function of the libration frequency $\hat{\omega}$ for $E=10^{-4}$ and $\epsilon=0.01$. The curve $B$ represents the kinetic energies computed from the linear numerical analysis while, on the curve, the square is from the analytical solution and the circles represent the result of the nonlinear numerical simulations. Apart from some small differences among the three different solutions, the scaled kinetic energies $\bar{E}_{k i n} / \epsilon^{2}$ are nearly independent of the libration frequency $\hat{\omega}$, and can be always expressed as $1 / 10$ plus or minus some small perturbations at any $E \ll 1$, reconfirming the theoretical prediction of the absence of resonance. In addition to the results for $E=10^{-4}$, we have also computed several solutions at $E=10^{-5}$. For example, the linear numerical analysis gives $\bar{E}_{k i n} / \epsilon^{2}=0.1095$ for $E=10^{-5}$ and $\epsilon=0.01$ at $\hat{\omega}=1.3093$ while the analytical result is $\bar{E}_{k i n} / \epsilon^{2}=0.1106$. When $\hat{\omega}=1.6604$, the linear numerical solution yields $\bar{E}_{k i n} / \epsilon^{2}=0.1012$ for $E=10^{-5}$ and $\epsilon=0.01$ with the analytical result is $\bar{E}_{k i n} / \epsilon^{2}=0.1019$. It reinforces the view that the scaled kinetic energy $\bar{E}_{k i n} / \epsilon^{2}$ to leading order is independent of the Ekman number $E$.

It is noteworthy that the higher inertial modes, because of their spatial complexity, are difficult to measure accurately in any experimental study. Whilst the pressure difference on the rotation axis may be accurately measured for the first mode $\hat{\omega}=1.3093$ (both figures 3 and 4 of Aldridge \& Toomre (1969)), the pressure difference for the mode with $\hat{\omega}=1.6604$ would be difficult to measure accurately. This can be immediately seen from the spatial variation of the three fundamental modes, $p_{12}, p_{13}$ and $p_{23}$, on the rotation axis, which is shown in figure 5 . It clearly 
illustrates that the pressure difference for the first fundamental mode $\left(\omega_{12}=1.3093\right)$ and the second mode $\left(\omega_{13}=0.9378\right)$ can be readily measured at the rotation axis either between the pole $(\theta=0, r=1)$ and the point $(\theta=0, r=1 / 2)$ or between the pole $(\theta=0, r=1)$ and the centre $(r=0)$. For the second fundamental mode $\left(\omega_{23}=1.6604\right)$, however, the pressure difference between the pole $(\theta=0, r=1)$ and the centre $(r=0)$, as depicted in figure 5 , will be difficult to measure in typical laboratory settings. This is why the difference between the pole $(\theta=0, r=1)$ and the point $(\theta=0, r=1 / 2)$ is shown in figure 4 .

Finally, the mean azimuthal flow $U(r, \theta)$ can be also obtained by performing the temporal integration over a period of libration

$$
U(r, \theta)=\frac{\hat{\omega}}{2 \pi}\left[\int_{0}^{2 \pi / \hat{\omega}} u_{\phi}(r, \theta, t) \mathrm{d} t\right],
$$

where $u_{\phi}(r, \theta, t)$ denotes the azimuthal component of a fully nonlinear numerical solution $\boldsymbol{u}$. Figure $6(a, b)$ unveils the profiles of the mean flow $U(r, \theta)$ in a meridional plane at two different librating frequencies, $\hat{\omega}=1.3093$ and $\hat{\omega}=1.6604$, for $E=10^{-4}$ and $\epsilon=0.01$ while figure $6(c)$ shows $U(r, \theta)$ as a function of $r$ at the equatorial plane $\theta=\pi / 2$. The mechanism of maintaining the mean flow $U(r, \theta)$ would be the combined effect of small viscosity and weak nonlinearity as discussed by Busse (2010) (see also Calkins et al. 2010; Sauret et al. 2010; Chan et al. 2011). Different profiles of $U(r, \theta)$ shown in figure 6 are attributable to different inertial modes that are excited by libration at different librating frequencies.

\section{Summary and some remarks}

We have studied an oscillatory fluid motion in a fluid-filled sphere forced by weak longitudinal libration, via three different but complementary methods: asymptotic analysis valid for $E \ll 1$, linear numerical analysis using a spectral method and nonlinear direct numerical simulation using a finite-element method. The explicitly analytical solution offers helpful insight into the problem, the linear numerical analysis is computationally inexpensive but does not give the pressure and the nonlinear numerical simulation is computationally expensive but yields directly both the flow velocity and the pressure. A satisfactory agreement is achieved among the three different solutions obtained via completely different ways when $E \leqslant 10^{-4}$.

Our theoretical and numerical results are consistent with the experimental observations (Aldridge \& Toomre 1969) but suggest a different theoretical interpretation. It is shown that the amplitude of the flow $\boldsymbol{u}$ forced by libration is nearly independent of the libration frequency $\hat{\omega}$ and, hence, that resonance cannot occur in the present problem. Thus, the amplitude of the librating flow in the present problem is always $|\boldsymbol{u}|=O(\epsilon)$ with its average kinetic energies $\bar{E}_{k i n}$ being given by $\epsilon^{2} / 10$ plus a small perturbation. In comparison, in other non-uniformly rotating problems (for example, Gans 1970; Meunier et al. 2008; Zhang et al. 2012), resonances do occur and the flow amplitude obeys $|\boldsymbol{u}|=O(\epsilon / \sqrt{E})$ at $E \ll 1$ when $\hat{\omega}=\omega_{n k}$ while $|\boldsymbol{u}|=O(\epsilon)$ away from $\omega_{n k}$. It is also found that the pressure difference amplitude along the axis of rotation reaches a sharp peak when $\hat{\omega}$ approaches a characteristic value $\omega_{n k}$, similar to that observed in previous experiments, but that the pressure difference in the equatorial plane is nearly independent of the libration frequency $\hat{\omega}$. Moreover, no other type of nonlinear resonances are observed in fully nonlinear, finite-element models in the weakly nonlinear regimes for $\epsilon=O\left(10^{-2}\right)$ with $10^{-3}<E \leqslant 10^{-5}$. It is the special 
(a)

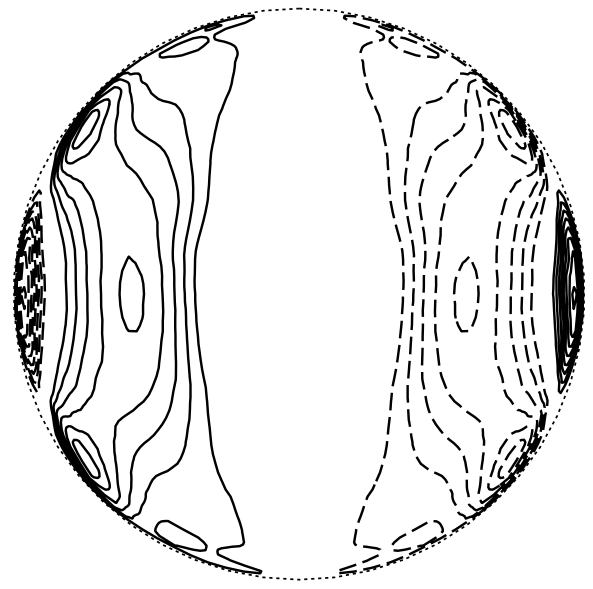

(b)

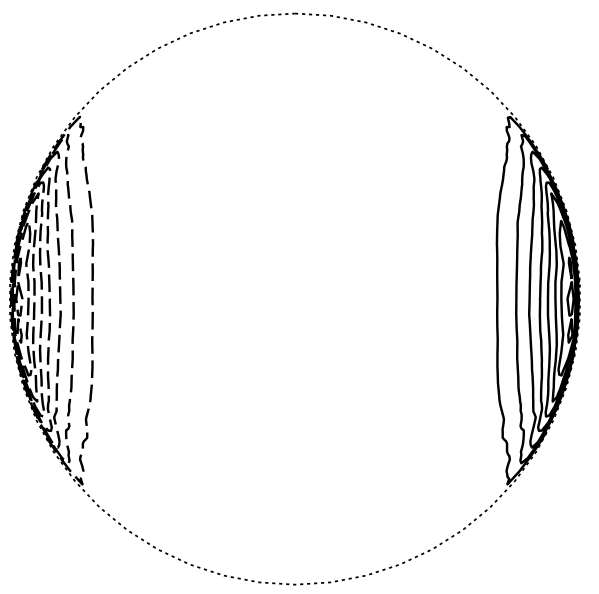

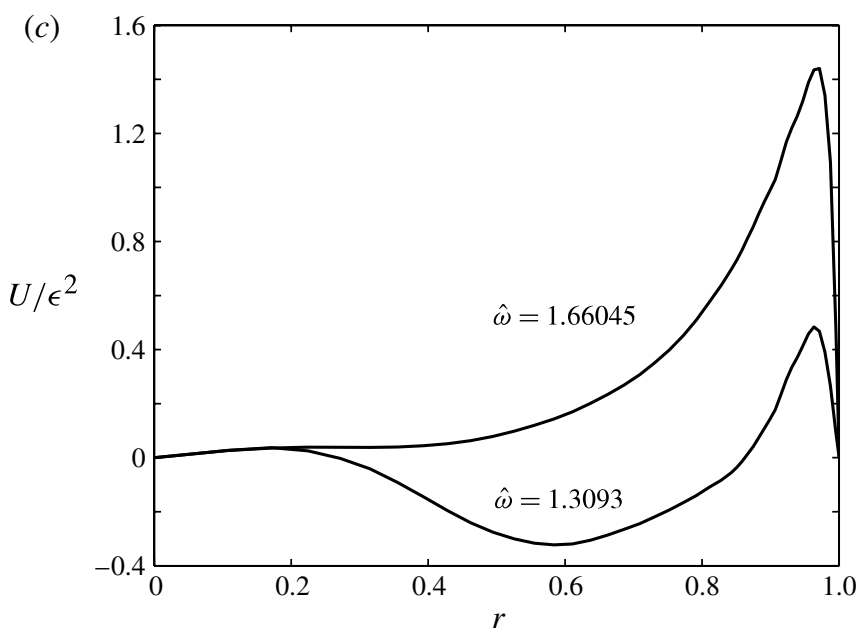

FIGURE 6. Contours of the mean zonal flow $U(r, \theta)$ in a meridional plane computed from nonlinear numerical solutions with $E=10^{-4}$ and $\epsilon=0.01$ for $(a) \hat{\omega}=1.3093$ and $(b)$ $\hat{\omega}=1.6604$. Solid contours are for $U(r, \theta)>0$ while dashed contours for $U(r, \theta)<0$. (c) The scaled mean flow $U(r, \pi / 2) / \epsilon^{2}$ plotted as a function of $r$ in the equatorial plane.

structure of the pressure solution, its dominant term vanishes at the rotating axis, that leads to a remarkable property: the pressure difference along the rotation axis correctly and accurately measures the weaker signal of a small secondary flow in the form of an oscillatory inertial mode. In short, the average kinetic energy $\bar{E}_{k i n} / \epsilon^{2}$ of the librating flow is, as shown curve B in figure 4, always given by

$$
\frac{\bar{E}_{k i n}}{\epsilon^{2}}=\frac{1}{10}+\text { small perturbations }
$$

at or near or away from a characteristic value $\omega_{n k}$ while the maximum pressure difference in the equatorial plane is, as shown curve $\mathrm{A}$ in figure 4 , always given by

$$
\frac{1}{\epsilon}\left|p\left(\theta=\frac{\pi}{2}, r=1, t\right)-p\left(\frac{\pi}{2}, r=0, t\right)\right|_{\max }=1+\text { small perturbations }
$$


at or near or away from a characteristic value $\omega_{n k}$ for $E \ll 1$. For librating spherical shells with a sizable inner core, the above conclusion needs to be changed quantitatively, but we expect that the main features such as the non-resonant response would remain unchanged.

Our findings agree with the axial pressure difference measurements made in previous studies (e.g. Aldridge \& Toomre 1969; Rieutord 1991). However, by using multiple solution methods, we show that longitudinal libration in a spherical fluid cavity is never resonant. This implies that longitudinal librating spherical systems, in particular, is not capable of generating strong geophysical or astrophysical turbulence. However, other librating systems are indeed capable of generating resonant, turbulent flows that are likely relevant to natural settings (e.g. Zhang et al. 2012).

\section{Acknowledgements}

We would like to thank Professor K. Aldridge for helpful discussions. K.Z. is supported by UK Leverhulme, STFC and NERC grants, K.H.C. is supported by Hong Kong RGC (grant number 700310), X.L. is supported by the NSFC (grant number 10633030) and CAS (grant number KZZD-EW-01-3) and J.A. is supported by the NSF Geophysics Program. The numerical computation is supported by Shanghai Supercomputer Center.

\section{REFERENCES}

Aldridge, K. D. \& Toomre, A. 1969 Axisymmetric inertial oscillations of a fluid in a rotating spherical container. J. Fluid Mech. 37, 307-323.

Busse, F. H. 1968 Steady fluid flow in a precessing spheroidal shell. J. Fluid Mech. 136, 739-751.

Busse, F. H. 2010 Mean zonal flows generated by librations of a rotating spherical cavity. J. Fluid Mech. 650, 505-512.

Calkins, M. A., Noir, J., Eldredge, J. \& Aurnou, J. M. 2010 Axisymmetric simulations of libration-driven fluid dynamics in a spherical shell geometry. Phys. Fluids 22, 086602.

CÉBron, D., Le BArs, M., NoIR, J. \& Aurnou, J. M. 2012 Libration driven elliptical instability. Phys. Fluids 24, 061703.

Chan, K., LiaO, X. \& ZHANG, K. 2011 Simulations of fluid motion in ellipsoidal planetary cores driven by longitudinal libration. Phys. Earth Planet. Inter. 187, 391-403.

ChAn, K., Zhang, K. \& LiAO, X. 2010 An EBE finite element method for simulating nonlinear flows in rotating spheroidal cavities. Intl J. Numer. Meth. Fluids 63, 395-414.

DERMOTT, S. F. 1979 Shapes and gravitational moments of satellites and asteroids. Icarus 37, $576-586$.

GANS, R. F. 1970 On the precession of a resonant cylinder. J. Fluid Mech. 41, 865-872.

Greenspan, H. P. 1964 On the transient motion of a contained rotating fluid. J. Fluid Mech. 21, 673-696.

Greenspan, H. P. 1968 The Theory of Rotating Fluids. Cambridge University Press.

Hollerbach, R. \& KeRswell, R. R. 1995 Oscillatory internal shear layers in rotating and precessing flows. J. Fluid Mech. 298, 327-339.

Kobine, J. J. 1995 Inertial wave dynamics in a rotating and precessing cylinder. J. Fluid Mech. 303, 233-252.

LIAO, X. \& ZHANG, K. 2012 On flow in weakly precessing cylinders: the general asymptotic solution. J. Fluid Mech. 709, 610-621.

MANASSEH, R. 1992 Breakdown regimes of inertia waves in a precessing cylinder. J. Fluid Mech. 243, 261-296.

Margot, J. L., Peale, S. J., Jurgens, R. F., Slade, M. A. \& Holin, I. V. 2007 Large longitude libration of mercury reveals a molten core. Science 316, 710-714. 
Meunier, P., Eloy, C., Lagrange, R. \& Nadal, 2008 A rotating fluid cylinder subject to weak precession. J. Fluid Mech. 599, 405-440.

Noir, J., Calkins, M. A., Lasbleis, M., Cantwell, J. \& Aurnou, J. M. 2010 Experimental study of libration-driven zonal flows in a straight cylinder. Phys. Earth Planet. Inter. 182, 98-106.

Noir, J., Cardin, P., Jault, D. \& Masson, J. P. 2003 Experimental evidence of nonlinear resonance effects between retrograde precession and the tilt-over mode within a spheroid. Geophys. J. Intl 154, 407-416.

Noir, J., CÉbron, D., Le Bars, M., Sauret, A. \& Aurnou, J. M. 2012 Experimental study of libration-driven zonal flows in non-axisymmetric containers. Phys. Earth Planet. Inter. 204-205, 1-10.

Noir, J., Hemmerlin, F., Wicht, J., BacA, S. M. \& Aurnou, J. M. 2009 An experimental and numerical study of librationally driven flow in planetary cores and subsurface oceans. Phys. Earth Planet. Inter. 173, 141-152.

RIEUTORD, M. 1991 Linear theory of rotating fluids using spherical harmonics part II, time-periodic flows. Geophys. Astrophys. Fluid Dyn. 59, 185-208.

Roberts, P. H. \& STEWARTSON, K. 1965 On the motion of a liquid in a spheroidal cavity of a precessing rigid body: II. Math. Proc. Cambridge Phil. Soc. 61, 279-288.

Sauret, A., Cebron, D., Morize, C. \& Le Bars, M. 2010 Experimental and numerical study of mean zonal flows generated by librations of a rotating spherical cavity. J. Fluid Mech. 662, 260-268.

Tilgner, A. 1999 Driven inertial oscillations in spherical shells. Phys. Rev. E 59, 1789-1794.

TILGNER, A. 2007 Rotational dynamics of the core. In Core Dynamics (ed. G. Schubert), Treatise of Geophysics, vol. 8, pp. 207-243. Elsevier.

Wood, W. W. 1966 An oscillatory disturbance of rigidly rotating fluid. Proc. R. Soc. Lond. A 293, $181-212$.

Zhang, K., Chan, K. \& Liao, X. 2011 On fluid motion in librating ellipsoids with moderate equatorial eccentricity. J. Fluid Mech. 673, 468-479.

Zhang, K., Chan, K. \& LiaO, X. 2012 Asymptotic theory of resonant flow in a spheroidal cavity driven by latitudinal libration. J. Fluid Mech. 692, 420-445.

Zhang, K., Earnshaw, P., Liao, X. \& Busse, F. H. 2001 On inertial waves in a rotating fluid sphere. J. Fluid Mech. 437, 103-119. 